\title{
The minimal model program for deformations of Hilbert schemes of points on the projective plane
}

\author{
Chunyi Li and Xiaolei Zhao
}

\begin{abstract}
We study the birational geometry of deformations of Hilbert schemes of points on $\mathbb{P}^{2}$. We show that moduli of Bridgeland-stable objects are smooth, irreducible, projective varieties, which are birationally equivalent to these deformations. Moreover, wall crossing in the space of Bridgeland-stability conditions induces the minimal model program for these deformations. In particular, for Hilbert schemes of points on $\mathbb{P}^{2}$, this proves a correspondence between destabilizing walls in the space of Bridgeland-stability conditions and stable base locus walls in the effective divisor cone, conjectured by Arcara, Bertram, Coskun and Huizenga.
\end{abstract}

\section{Introduction}

The Hilbert scheme of points on an algebraic variety is the moduli space that parameterizes 0 -dimensional subschemes of length $n$ on the variety, with given positive integer $n$. In the case of curves, the Hilbert scheme of points is isomorphic to the symmetric product of the curve itself. When the variety has dimension at least 3, the Hilbert scheme of points may have wild singularities. In the surface case, the Hilbert scheme of points is smooth and connected, and has many beautiful geometric properties. The Hilbert scheme also parameterizes ideal sheaves with trivial first Chern class and a given second Chern class, so can be viewed as a moduli space of sheaves on the surface. The goal of this paper is to understand the birational geometry of Hilbert schemes on the projective plane and their deformations. This fits into a general program to study the birational geometry of moduli spaces. In the case of moduli of curves, this is known as the Hassett-Keel program and much research has been done. In the case of moduli spaces of sheaves, recent progress has been made via Bridgeland-stability conditions.

The notion of stability conditions on a triangulated category $T$ has been introduced by Bridgeland in [Bri07]. It is given by abstracting the usual properties of the $\mu$-stability for sheaves on projective varieties. The central charge, which substitutes the slope $\mu$, is a group homomorphism from the numerical Grothendieck group to $\mathbb{C}$ and satisfies several conditions, including the existence of Harder-Narasimhan filtrations. Bridgeland-stability conditions form a natural topological space $\operatorname{Stab}(T)$, which becomes a complex manifold of dimension that of the numerical Grothendieck group.

In general, it is a very difficult problem to construct Bridgeland-stability conditions on a given

Received 22 February 2016, accepted in final form 27 April 2017.

2010 Mathematics Subject Classification 14D20, 14E30.

Keywords: birational geometry, stability condition, geometric invariant theory.

This journal is (c) Foundation Compositio Mathematica 2018. This article is distributed with Open Access under the terms of the Creative Commons Attribution Non-Commercial License, which permits non-commercial reuse, distribution, and reproduction in any medium, provided that the original work is properly cited. For commercial re-use, please contact the Foundation Compositio Mathematica. 


\section{MMP FOR DEFORMED HiLb $\mathbb{P}^{2}$}

triangulated category. When $T$ is the bounded derived category of coherent sheaves on a smooth surface $X$, the existence is established in [AB13, Bri08]. We now focus on this surface case. Given a numerical class $v$ and a stability condition $\sigma$ on $D^{b}(\operatorname{Coh}(X))$, we can consider the moduli space $\mathfrak{M}_{\sigma}(v)$ of $\sigma$-stable objects of numerical class $v$. Two natural questions on $\mathfrak{M}_{\sigma}(v)$ arise:

(1) When is $\mathfrak{M}_{\sigma}(v)$ a good geometric object to study?

(2) When $\sigma$ changes in $\operatorname{Stab}\left(D^{b}(X)\right)$, what is the behavior of $\mathfrak{M}_{\sigma}(v)$ ?

For a general smooth surface, the known answers to both questions are mostly vague. For the first question, there are a few ways to determine when $\mathfrak{M}_{\sigma}(v)$ is projective. For the second question, ideally, the stability space has a well-behaved chamber structure. In each chamber, we have $\mathfrak{M}_{\sigma}(v) \simeq \mathfrak{M}_{\sigma^{\prime}}(v)$. Between different chambers, there exists a birational map $\mathfrak{M}_{\sigma}(v) \rightarrow$ $\mathfrak{M}_{\sigma^{\prime}}(v)$. Yet, the ideal picture is far from being complete. It is only set up or partially (or conjecturally) set up when $X$ is a $\mathrm{K} 3$ surface, the projective plane [ABCH13], a high-degree del Pezzo surface [BC13], a Hirzebruch surface [BC13], an Enrique surface [Nue16] or an abelian surface [MYY11, MYY14]. One of the most successful cases is Bayer and Macrì's work [BM14a, BM14b] on K3 surfaces. The authors construct nef divisors on the moduli spaces of Bridgelandstable objects and complete the wall-crossing picture.

Let $\mathfrak{M}_{\sigma}(n)$ be the moduli space of $\sigma$-stable objects on $\mathbb{P}^{2}$ with numerical type $\left(r, c_{1}, \chi\right)=$ $(1,0,1-n)$, where we mean their numerical type as Hilbert schemes. In [ABCH13], the authors studied the two questions in this case. They describe a wall and chamber structure on $\operatorname{Stab}\left(D^{b}\left(\mathbb{P}^{2}\right)\right)$ for the invariant $\left(r, c_{1}, \chi\right)=(1,0,1-n)$. On a particular upper half-plane slice of $\operatorname{Stab}\left(D^{b}\left(\mathbb{P}^{2}\right)\right)$, the walls are a sequence of nested semicircles in each quadrant, plus the vertical axis. For certain $\sigma$ in the second quadrant, they show $\mathfrak{M}_{\sigma}(n) \simeq \operatorname{Hilb}^{n} \mathbb{P}^{2}$. By choosing certain representative stability conditions in each chamber, the authors prove that there are finitely many chambers for which $\mathfrak{M}_{\sigma}(n)$ is non-empty and, in this case, projective. Moreover, for small values $n \leqslant 9$, the authors also write down an explicit correspondence between the destabilizing walls in the stability space and the base locus decomposition walls of the effective cone (in the sense of the minimal model program (MMP)). For general values of $n$, the explicit formula for this correspondence remains conjectural. One difficulty is to get a better answer to the first question above, in other words, to control the behavior of $\mathfrak{M}_{\sigma}(n)$, especially the smoothness and irreducibility. In this paper, we solve these two questions in the case of $\mathbb{P}^{2}$ with numerical type $\left(r, c_{1}, \chi\right)=(1,0,1-n)$.

TheOREM 1.1 (Theorem 4.11). Adopting the notation as above, we have the following statements:

(i) When $\sigma$ is not on any wall, $\mathfrak{M}_{\sigma}(n)$ is either a smooth, projective, irreducible variety of dimension $2 n$, or empty.

(ii) Given $\sigma$ and $\sigma^{\prime}$ not on any wall, $\mathfrak{M}_{\sigma}(n)$ and $\mathfrak{M}_{\sigma^{\prime}}(n)$ are birationally equivalent to each other when they are both non-empty.

To prove this theorem, we study the geometric invariant theory (GIT) construction of $\mathfrak{M}_{\sigma}(n)$ in detail and control the dimension of the exceptional locus for each birational map associated with the wall crossing. Then, for each moduli space $\mathfrak{M}_{\sigma}(n)$, we assign an ample line bundle on it. Applying the variation of GIT in [DH98] and [Tha96], we show that a Bridgeland-stability wall crossing of $\mathfrak{M}_{\sigma}(n)$ is the flip with respect to the line bundle. As a result, the nested semicircular walls are in one-to-one correspondence with the stable base locus decomposition walls of the effective divisor cone of $\operatorname{Hilb}^{n}\left(\mathbb{P}^{2}\right)$. In addition, given the location of the destabilizing wall, its corresponding base locus decomposition wall is given by an explicit formula in Proposition 5.7. 


\section{Li AND X. ZhaO}

Note that in certain cases, the correspondence of the 'last wall' has been established in the paper [CHW17] by Coskun, Huizenga and Woolf. Recall that the (rational) Picard group of $\mathbb{P}^{2}$ is spanned by divisors $\Delta$ and $H$, where $\Delta$ is the exceptional divisor of the Hilbert-Chow morphism to $\mathrm{Sym}^{n} \mathbb{P}^{2}$ and $H$ is the pull-back of $\mathcal{O}(1)$ on $\mathrm{Sym}^{n} \mathbb{P}^{2}$ via this morphism.

Theorem 1.2 (Theorem 5.5 and Proposition 5.7). When the stability condition varies in the second quadrant of the upper half-plane parameterizing Bridgeland-stability conditions on $D^{b}\left(\mathbb{P}^{2}\right)$, the corresponding moduli space of stable objects performs birational transformations, and this wall-crossing process is equivalent to the minimal model program for Hilb ${ }^{n} \mathbb{P}^{2}$.

This gives a one-to-one correspondence between the destabilizing walls in the upper half-plane of stability conditions and the stable base locus walls in the effective divisor cone. In particular, the destabilizing semicircular wall with center $-m-\frac{3}{2}$ corresponds to the stable base locus wall spanned by the divisor $m H-\frac{1}{2} \Delta$.

Another important aim of this paper is to extend this theory to deformations of $\mathrm{Hilb}^{n} \mathbb{P}^{2}$ by methods from non-commutative algebraic geometry. Here, we use the notion of Sklyanin algebras $S=\operatorname{Skl}(E, \mathcal{L}, \lambda)$, which are non-commutative deformations of the homogeneous coordinate ring of $\mathbb{P}^{2}$. Such a Sklyanin algebra depends on a cubic curve $E$ on $\mathbb{P}^{2}$, an automorphism $\lambda$ of $E$ and a degree 3 line bundle on $E$. The foundation of this non-commutative theory has been set up in [ATVdB90, ATVdB91, AVdB90, NS07, SvdB01]. For these non-commutative $\mathbb{P}^{2}$, we can still construct moduli spaces $\mathfrak{M}_{\mathrm{GM}}^{s s}(1,0,1-n)$, which turn out to be smooth projective varieties (in the ordinary commutative sense!) and are in fact deformations of $\mathrm{Hilb}^{n} \mathbb{P}^{2}$ by [Hit12] and [NS07]. We will write these deformations as $\mathrm{Hilb}^{n} S$.

We study Bridgeland-stability conditions on $D^{b}(\operatorname{Coh}(S))$, which are very similar to those for $\mathbb{P}^{2}$. In particular, there is a similar chamber structure on the upper half-plane slice of the Bridgeland space, and Theorem 1.2 also holds for $\mathfrak{M}_{\sigma}^{s s}(n)$ associated with non-commutative $\mathbb{P}^{2}$. However, the behavior of wall crossing over the vertical wall is different in this case, and this changes the correspondence between the walls of stability space and the base locus decomposition walls of the effective cone. In this case, we have the following theorem.

Theorem 1.3 (Theorems 5.13 and 5.5). For a deformation of $\operatorname{Hilb}^{n} \mathbb{P}^{2}$ with $n \geqslant 3$, the vertical wall induces an involution, and stable base locus walls are in one-to-one correspondence with semicircular destabilizing walls in both the first and the second quadrants of the upper half-plane of Bridgeland-stability conditions. In particular, the effective cone of $\mathrm{Hilb}^{n} S$ has a symmetric stable base locus decomposition, as shown in the diagram below.

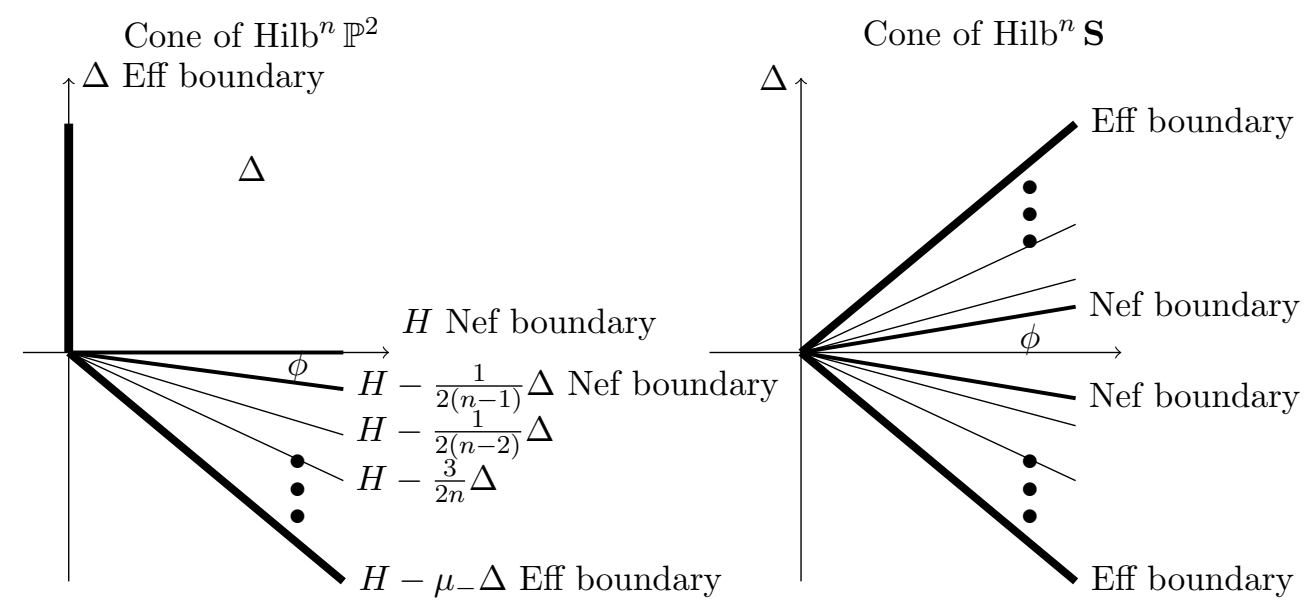




\section{MMP FOR DEFORMED HILB $\mathbb{P}^{2}$}

In addition, this map is 'monotone' in the sense that the two most-inner walls on the two quadrants correspond to the two edges of the effective cone, respectively. When one moves from inner semicircles to the outside, the corresponding stable base locus wall moves in one direction. This reveals a symmetric structure of the Mori decomposition of the effective divisor cone of Hilb ${ }^{n} S$. Diagrams of divisor cones of $\operatorname{Hilb}^{n} \mathbb{P}^{2}$ and a generic Hilb ${ }^{n} \mathbf{S}$ are shown above.

In the left picture, $\Delta$ is the exceptional divisor of the Hilbert-Chow map to $\operatorname{Sym}^{n} \mathbb{P}^{2}$, and $H$ is the pull-back of $\mathcal{O}(1)$ on $\operatorname{Sym}^{n} \mathbb{P}^{2}$. The picture on the right is for Hilb ${ }^{n} \mathbf{S}$. Here, $\Delta$ and $H$ are the corresponding divisor classes under deformation.

After we obtained the results in this paper, but before we finished writing it, the paper [CH14] of Coskun and Huizenga appeared. In [CH14], the authors obtained the 'correspondence of walls' result for Hilb ${ }^{n} \mathbb{P}^{2}$ in certain cases. The paper [CH14] does not treat the case of Hilb $^{n} \mathbf{S}$, which is new in this paper. Also, in [CH14] the authors study the 0-dimensional monomial subschemes $Z$ of $\mathbb{P}^{2}$ when $\mathcal{I}_{Z}$ is destabilized to get their result. Our approach is quite different, and the approach in [CH14] does not apply to the non-commutative case; for example, a general point in Hilb $^{n} S$ does not correspond to an ideal sheaf on $S$. We show the smoothness and irreducibility of each moduli space by showing some Ext $^{2}$ vanishing. These good properties allow one to apply the variation of geometric invariant theory to obtain the correspondence.

\section{Background material}

In Section 2.1, we recall the definition of Sklyanin algebras and how to use them to construct deformations of Hilbert schemes of points on $\mathbb{P}^{2}$. In Section 2.2, we recall the definition of Bridgeland-stability conditions and the construction of stability conditions on the category of sheaves over Sklyanin algebras.

\subsection{Review: Sklyanin algebras and deformations of Hilbert schemes of points on $\mathbb{P}^{2}$}

For each positive integer $n$, the Hilbert scheme of $n$ points on $\mathbb{P}^{2}$ admits a 2-dimensional deformation family. A generic deformation in this family can be constructed as a certain moduli space over the non-commutative projective plane given by a Sklyanin algebra. In this subsection, we want to recall this construction from [NS07] by Nevins and Stafford. We will start with the definition (Definition 2.1) and some properties of the Sklyanin algebras from non-commutative algebraic geometry; further details can be found in [ATVdB90, ATVdB91, AVdB90, NS07]. The main construction is summarized in Theorem 2.4.

Let $\iota: E \hookrightarrow \mathbb{P}^{2}$ be a smooth elliptic curve embedded in the projective space. Fix the line bundle $\mathcal{L}=\iota^{*}\left(\mathcal{O}_{\mathbb{P}^{2}}(1)\right)$ of degree 3 and an automorphism $\lambda \in \operatorname{Aut}(E)$ which is given by a translation under the group structure; we denote the graph of $\lambda$ by $\Gamma_{\lambda} \subset E \times E$. Let $V:=\mathrm{H}^{0}(E, \mathcal{L})$; then we have a 3 -dimensional subspace $\mathcal{R}=\mathcal{R}(E, \mathcal{L}, \lambda)$ in $V \otimes V$ given by

$$
\mathcal{R}:=\mathrm{H}^{0}\left(E \times E,(\mathcal{L} \otimes \mathcal{L})\left(-\Gamma_{\lambda}\right)\right) \subset \mathrm{H}^{0}(E \times E, \mathcal{L} \otimes \mathcal{L})=V \otimes V .
$$

Definition 2.1 ([ATVdB90]). The 3-dimensional Sklyanin algebra is the algebra

$$
S=\operatorname{Skl}(E, \mathcal{L}, \lambda)=T(V) /(\mathcal{R}),
$$

where $T(V)$ denotes the tensor algebra of $V$ and $(\mathcal{R})$ is the two-sided ideal generated by $\mathcal{R}$.

In the special case when $\lambda$ is the identity, $\operatorname{Skl}(E, \mathcal{L}, \mathrm{Id})$ is just the commutative polynomial ring $\mathbb{C}[x, y, z]$. In general, $\operatorname{Skl}(E, \mathcal{L}, \lambda)$ can be written as a non-commutative $\mathbb{C}$-algebra with 


\section{Li AND X. ZhaO}

generators $x_{1}, x_{2}, x_{3}$ and relations

$$
a x_{i} x_{i+1}+b x_{i+1} x_{i}+c x_{i+2}^{2}=0, \quad i=1,2,3 \bmod 3,
$$

where $a, b, c \in \mathbb{C}^{*}$ are such that $(3 a b c)^{3} \neq\left(a^{3}+b^{3}+c^{3}\right)^{3}$.

The Sklyanin algebra $S$ is a graded algebra with grading induced from the tensor algebra $T(V)$. Let Mod- $S$ be the category of right $S$-modules. Also, let $\mathrm{Gr}-S$ be the category of graded right $S$-modules, with homomorphisms $\operatorname{Hom}_{S}(M, N)$ consisting of graded homomorphisms of degree 0 . For a graded module $M=\oplus_{i \in \mathbb{Z}} M_{i}$, the shifted module $M(n)$ is defined to be the graded module with $M(n)_{i}=M_{i+n}$ for all $i$.

Since $S$ is Noetherian [NS07, Lemma 5.1], we can consider the subcategory gr- $S$ of Noetherian objects in Gr- $S$. A module $M \in \operatorname{gr}-S$ is called right bounded if $M_{i}=0$ for $i \gg 0$. The full Serre subcategory of gr- $S$ generated by the right-bounded modules is denoted by rb- $S$ and the quotient category by qgr- $S:=\operatorname{gr}-S / \mathrm{rb}-S$. One has a pair of adjoint functors $\pi: \operatorname{gr}-S \leftrightarrows \operatorname{qgr}-S: \Gamma^{*}$, where $\pi$ is the natural projection and its adjoint $\Gamma^{*}$ is the 'global section' functor. When $S \simeq \mathbb{C}[x, y, z]$, the quotient qgr- $S$ is equivalent to the category of coherent sheaves on Proj $\mathbb{C}[x, y, z]$. For this reason, we call an object $\mathcal{M} \in$ qgr- $S$ a sheaf on $S$. In particular, we will use $\mathcal{O}_{S}$ to denote $S$ itself.

As in the commutative case, we can define several numerical invariants for sheaves on $S$. A sheaf $\mathcal{M}$ on $S$ is called torsion if each element in $\Gamma^{*}(\mathcal{M})$ is annihilated by a non-zero element of $S$ and torsion free if no element is so. A torsion-free $\mathcal{M}$ is of $\operatorname{rank} r$ if $\mathcal{M}$ contains a direct sum of $r$, but not $r+1$, non-zero submodules. In general, a sheaf $\mathcal{M}$ has a canonical maximal torsion subsheaf, whose quotient is torsion free, and the rank of $\mathcal{M}$ is defined to be the rank of this quotient. We use $\operatorname{rk}(\mathcal{M})$ to denote the $\operatorname{rank}$ of $\mathcal{M}$.

The first Chern class $c_{1}(\mathcal{M})$ is defined in [NS07, Lemma 3.7] as the unique function $c_{1}:$ qgr- $S$ $\rightarrow \mathbb{Z}$ with the following properties:

- $c_{1}$ is additive on short exact sequences;

$-c_{1}\left(\mathcal{O}_{S}(m)\right)=m$ for all $m \in \mathbb{Z}$.

The Euler character on the category qgr- $S$ is defined as usual:

$$
\chi(\mathcal{E}, \mathcal{F}):=\sum_{i}(-1)^{i} \operatorname{dim} \operatorname{Ext}^{i}(\mathcal{E}, \mathcal{F})
$$

for $\mathcal{E}, \mathcal{F} \in \operatorname{qgr}-S$. For a sheaf $\mathcal{M}$, we set $\chi(\mathcal{M}):=\chi(\mathcal{O}, \mathcal{M})$. The Hilbert polynomial of $\mathcal{M}$ is the polynomial $p_{\mathcal{M}}(t):=\chi(\mathcal{M}(t))$. The slope of $\mathcal{M}$ is defined as $\mu^{\mathrm{GM}}(\mathcal{M}):=c_{1}(\mathcal{M}) / \operatorname{rk}(\mathcal{M})$. A torsion-free sheaf $\mathcal{M}$ is called slope stable (respectively, slope semistable), if for every non-zero proper submodule $\mathcal{F} \subset \mathcal{M}$, one has

$$
\mu^{\mathrm{GM}}(\mathcal{F})<\mu^{\mathrm{GM}}(\mathcal{M} / \mathcal{F}) \quad\left(\text { respectively, } \mu^{\mathrm{GM}}(\mathcal{F}) \leqslant \mu^{\mathrm{GM}}(\mathcal{M} / \mathcal{F})\right) .
$$

Given a torsion-free sheaf $\mathcal{M}$, there exists the Harder-Narasimhan filtration

$$
0=\mathcal{M}_{0} \subset \mathcal{M}_{1} \subset \cdots \subset \mathcal{M}_{n}=\mathcal{M}
$$

such that each quotient $\mathcal{F}_{i}=\mathcal{M}_{i} / \mathcal{M}_{i-1}$ is slope semistable and $\mu^{\mathrm{GM}}\left(\mathcal{F}_{i}\right)>\mu^{\mathrm{GM}}\left(\mathcal{F}_{i+1}\right)$. We write $\mu_{+}^{\mathrm{GM}}(\mathcal{M})$ for $\mu^{\mathrm{GM}}\left(\mathcal{F}_{1}\right)$ and $\mu_{-}^{\mathrm{GM}}(\mathcal{M})$ for $\mu^{\mathrm{GM}}\left(\mathcal{F}_{n}\right)$. Moreover, we formally define the second Chern character $\operatorname{ch}_{2}(\mathcal{M})$ by

$$
\operatorname{ch}_{2}(\mathcal{M}):=\chi(\mathcal{M})-\frac{3}{2} c_{1}(\mathcal{M})-\operatorname{rk}(\mathcal{M})
$$




\section{MMP FOR DEFORMED HiLb $\mathbb{P}^{2}$}

Next, we will state two results on sheaves in $\operatorname{qgr}-S$. For sheaves on the projective plane, these are standard results. For the benefit of readers mainly interested in the non-deformed case, we postpone the proofs to Appendix 5.3.

Lemma 2.2. Let $\mathcal{M} \in$ qgr- $S$ be a sheaf. Then:

(i) We have $c_{1}(\mathcal{M}(s))=c_{1}(\mathcal{M})+s \cdot \operatorname{rk}(\mathcal{M})$ for any $s \in \mathbb{Z}$.

(ii) If $\mathcal{M}$ is torsion and non-zero, then $c_{1}(\mathcal{M}) \geqslant 0$; if in addition $c_{1}(\mathcal{M})=0$, then $\chi(\mathcal{M})>0$.

Let $D^{b}$ (qgr- $S$ ) be the bounded derived category of qgr- $S$. We rephrase one of the main results in [NS07], which is a non-commutative analog of the description of $D^{b}\left(\mathbb{P}^{2}\right)$ [Beř83].

Proposition 2.3 ([NS07, Proposition 6.20]). The derived category $D^{b}$ (qgr- $S$ ) is generated by (that is, is the closure under extension and homological shift of) $\mathcal{O}(k-1), \mathcal{O}(k)$, and $\mathcal{O}(k+1)$ for any $k \in \mathbb{Z}$.

As a consequence, the invariants $\{$ rank, first Chern class, Euler character $\}$ generate the numerical Grothendieck group of $D^{b}(\operatorname{qgr}-S)$. The importance of Sklyanin algebras is shown in the following theorem in [NS07]. There, the authors prove that deformations of $\mathrm{Hilb}^{n} \mathbb{P}^{2}$ can be constructed as the moduli spaces of semistable sheaves in qgr- $S$ with numerical invariants $(1,0,1-n)$. As pointed out in [Hit12], the generic deformation of $\mathrm{Hilb}^{n} \mathbb{P}^{2}$ can be constructed in this way.

ThEOREM 2.4 ([NS07, Theorems 8.11 and 8.12]). Let $\mathcal{B}$ be a smooth curve defined over $\mathbb{C}$, and let $S_{\mathcal{B}}\left(=S_{\mathcal{B}}(E, \mathcal{L}, \lambda)\right)$ be a flat family of algebras such that $S_{p} \cong \mathbb{C}[x, y, z]$ for some point $p \in \mathcal{B}$. Let $S_{b}$ be the algebra over the point $b \in \mathcal{B}$. Then, there exists a projective variety $\mathfrak{M}_{\mathcal{B}}^{s s}(1,0,1-n)$, smooth over $\mathcal{B}$ and such that $\mathfrak{M}_{\mathcal{B}}^{s s}(1,0,1-n) \otimes_{\mathcal{B}} \mathbb{C}(b)=\mathfrak{M}_{S_{b}}^{s s}(1,0,1-n)$ and each $\mathfrak{M}_{S}^{s s}(1,0,1-n)$ is a smooth, projective, fine moduli space for equivalence classes of rank 1 torsion-free modules $\mathcal{M} \in \operatorname{qgr}-S$ with $c_{1}(\mathcal{M})=0$ and $\chi(\mathcal{M})=1-n$. In particular, $\mathfrak{M}_{S_{p}}^{s s}(1,0,1-n)$ is isomorphic to Hilb $^{n} \mathbb{P}^{2}$, and each $\mathfrak{M}_{S_{b}}^{s s}(1,0,1-n)$ is a deformation of $\mathrm{Hilb}^{n} \mathbb{P}^{2}$.

We will write $\operatorname{Hilb}^{n} \mathbf{S}$ instead of $\mathfrak{M}_{S}^{s s}(1,0,1-n)$ for short.

Proposition 2.5. The Picard number of $\mathrm{Hilb}^{n} \mathbf{S}$ is 2 .

Proof. By the formula on the second page of [Nak97] by Nakajima, $b_{2}\left(\operatorname{Hilb}^{n} \mathbf{S}\right)=b_{2}\left(\operatorname{Hilb}^{n} \mathbb{P}^{2}\right)=2$. Since $\mathrm{Hilb}^{n} \mathbf{S}$ is projective and the Hodge numbers satisfy $h^{1,1} \geqslant 1$ and $h^{0,2}=h^{2,0}$, one must have $h^{1,1}=2$.

\subsection{Review: Bridgeland-stability conditions on $D^{b}(\mathrm{qgr}-S)$}

The Bridgeland-stability conditions are introduced in [Bri07]. In this section, we will first recall the basic definition of the Bridgeland-stability conditions in general, and then work out the constructions and properties of the Bridgeland-stability conditions on $D^{b}$ (qgr- $S$ ).

Let $K($ qgr- $S)$ be the numerical Grothendieck group of $D^{b}$ (qgr- $\left.S\right)$, in other words, the free abelian group generated by $r, c_{1}$ and $\chi$.

Definition 2.6. A pre-stability condition $\sigma$ on $D^{b}(\operatorname{qgr}-S)$ is a pair $(Z, \mathcal{A})$, where

$$
Z: K(\operatorname{qgr}-S) \rightarrow \mathbb{C}
$$

is a group homomorphism and $\mathcal{A} \subset D^{b}(\operatorname{qgr}-S)$ is the heart of a bounded t-structure, such that the following conditions hold: 


\section{Li And X. ZhaO}

(i) For any non-zero object $E \in \mathcal{A}$, we have

$$
Z(E) \in\left\{r e^{i \phi \pi}: r>0, \phi \in(0,1]\right\} .
$$

(ii) Harder-Narasimhan property: for any $E \in \mathcal{A}$, there is a filtration of finite length in $\mathcal{A}$,

$$
0=E_{0} \subset E_{1} \subset \cdots \subset E_{n}=E,
$$

such that each subquotient $F_{i}=E_{i} / E_{i-1}$ is $Z$-semistable with $\phi\left(F_{i}\right)>\phi\left(F_{i+1}\right)$, where the phase $\phi=\frac{1}{\pi} \operatorname{Arg}(Z)$ is in $(0,1]$.

Here an object $E \in \mathcal{A}$ is said to be $Z$-stable (respectively, $Z$-semistable) if for any subobject $0 \neq F \subsetneq E$ with $F \in \mathcal{A}$, we have

$$
\phi(F)<\phi(E) \quad \text { (respectively, } \phi(F) \leqslant \phi(E) .
$$

The group homomorphism $Z$ is called the central charge of the stability condition.

Definition 2.7 ([KS08, Section 2.1], [BMS16, Appendix A], [MS16]). The pre-stability condition $\sigma=(Z, \mathcal{A})$ satisfies the support property if there exists a quadratic form $Q$ on the vector space $K($ qgr- $S) \otimes \mathbb{R}$ such that

- the kernel of $Z$ is negative definite with respect to $Q$, and

- for any $\sigma$-semistable object $E \in \mathcal{A}$, we have $Q(v(E)) \geqslant 0$.

Here $v$ maps $E$ to its numerical Grothendieck group. A pre-stability condition is a stability condition if it satisfies the support condition.

The rest of this section is devoted to the construction of stability conditions on $D^{b}$ (qgr- $S$ ). First, we recall the notion of torsion pairs, which is essential to constructing t-structures. A pair of full subcategories $(\mathcal{F}, \mathcal{T})$ of qgr-S is called a torsion pair if it satisfies the following two conditions:

(1) For all $F \in \operatorname{obj} \mathcal{F}$ and $T \in \operatorname{obj} \mathcal{T}$, we have $\operatorname{Hom}(T, F)=0$.

(2) Each sheaf $E$ in qgr- $S$ fits in a short exact sequence

$$
0 \rightarrow T \rightarrow E \rightarrow F \rightarrow 0,
$$

where $T \in \operatorname{obj} \mathcal{T}$ and $F \in \operatorname{obj} \mathcal{F}$. In addition, the extension class in $\operatorname{Ext}^{1}(F, T)$ is uniquely determined up to isomorphism.

A torsion pair defines a t-structure on $D^{b}($ qgr- $S)$ by

$$
\begin{aligned}
& \mathcal{D}^{\geqslant 0}=\left\{C^{\bullet} \mid \mathrm{H}^{-1}\left(C^{\bullet}\right) \in \mathcal{F} \text { and } \mathrm{H}^{i}\left(C^{\bullet}\right)=0 \text { for } i<-1\right\}, \\
& \mathcal{D}^{\leqslant 0}=\left\{C^{\bullet} \mid \mathrm{H}^{0}\left(C^{\bullet}\right) \in \mathcal{T} \text { and } \mathrm{H}^{i}\left(C^{\bullet}\right)=0 \text { for } i>0\right\} .
\end{aligned}
$$

As in the $\mathbb{P}^{2}$ case, given $s \in \mathbb{R}$, we can define full subcategories $\mathrm{Coh}_{>s}$ and $\mathrm{Coh}_{\leqslant s}$ of qgr- $S$ as follows:

$-T \in \mathrm{Coh}_{>s}$ if $T$ is torsion or $\mu_{-}\left(T^{\prime}\right)>s$, where $T^{\prime}$ is the torsion-free quotient of $T$;

$-F \in \mathrm{Coh}_{\leqslant s}$ if $F$ is torsion free and $\mu_{+}(F) \leqslant s$.

By [Bri08, Lemma 6.1], the pair $\left(\mathrm{Coh}_{\leqslant s}, \mathrm{Coh}_{>s}\right)$ is a torsion pair. Let $\mathrm{Coh}_{\# s}$ be the heart of the t-structure determined by the torsion pair $\left(\mathrm{Coh}_{\leqslant s}, \mathrm{Coh}_{>s}\right)$; we may define a central charge $Z_{s, t}=-d_{s, t}+i r_{s, t}$ depending on a parameter $t>0$ by

$$
\begin{aligned}
& r_{s, t}(E):=\left(c_{1}-r s\right) t \\
& d_{s, t}(E):=-r t^{2} / 2+\left(s^{2} / 2-1\right) r-(3 / 2+s) c_{1}+\chi .
\end{aligned}
$$




\section{MMP FOR DEFORMED HILB $\mathbb{P}^{2}$}

We may write $\mu_{s, t}=d_{s, t} / r_{s, t}$ as the tilt slope of an object in $\mathrm{Coh}_{\# s}$.

For $\mathbb{P}^{2}$, the pair $\left(Z_{s, t}, \mathrm{Coh}_{\# s}\right)$ is a stability condition on $D^{b}\left(\mathbb{P}^{2}\right)$ by [AB13]. The following proposition says that this construction also works for $D^{b}(\operatorname{qgr}-S)$. The proof is a little involved and is given in Appendix 5.3. The main issue is that several tools in the $\mathbb{P}^{2}$ case are missing in this deformed case.

Proposition 2.8. Adopt the notation as above. Then $\left(Z_{s, t}, \mathrm{Coh}_{\# s}\right)$ is a stability condition on $D^{b}$ (qgr- $S$ ). Moreover, the map $\mathbb{R}_{>0} \times \mathbb{R} \rightarrow \operatorname{Stab}($ qgr- $S$ ) is continuous.

Let $\mathcal{A}(k)$ be the extension closure of $\mathcal{O}(k-1)[2], \mathcal{O}(k)[1]$ and $\mathcal{O}(k+1)$. Since $\{\mathcal{O}(k-1)$, $\mathcal{O}(k), \mathcal{O}(k+1)\}$ is a full strong exceptional collection by Proposition 2.3, $\mathcal{A}(k)$ is the heart of a t-structure on $D^{b}(\mathrm{qgr}-S)$; see [Mac07, Lemma 3.16]. Objects in $\mathcal{A}(k)$ are of the form

$$
\mathcal{O}(k-1) \otimes \mathbb{C}^{n_{-1}} \rightarrow \mathcal{O}(k) \otimes \mathbb{C}^{n_{0}} \rightarrow \mathcal{O}(k+1) \otimes \mathbb{C}^{n_{1}},
$$

where $n_{-1}, n_{0}, n_{1}$ are some non-negative integers. We write $\vec{n}=\left(n_{-1}, n_{0}, n_{1}\right)$ and call $\vec{n}$ the dimension vector of the object. One may construct a central charge $Z$ for $\mathcal{A}(k)$ by letting $Z(\mathcal{O}(k-1)[2])=z_{-1}, Z(\mathcal{O}(k)[1])=z_{0}$ and $Z(\mathcal{O}(k+1))=z_{1}$ for any collection of complex numbers $z_{i}$ on the upper half-plane $\left\{r e^{i \phi \pi} \mid r>0, \phi \in(0,1]\right\}$. The pair $(Z, \mathcal{A}(k))$ is a stability condition on $D^{b}(\operatorname{qgr}-S)$.

EXAMPLE 2.9. By [NS07, Theorem 5.6], any torsion-free sheaf with numerical class $\left(r k, c_{1}, \chi\right)=$ $(1,0,1-n)$ can always be written as the middle cohomology sheaf of the complex

$$
\mathcal{O}(-1) \otimes \mathbb{C}^{n} \rightarrow \mathcal{O} \otimes \mathbb{C}^{2 n+1} \rightarrow \mathcal{O}(1) \otimes \mathbb{C}^{n}
$$

Note that in the case of $\mathbb{P}^{2}$, these sheaves correspond to the ideal sheaves of subschemes of length $n$.

\section{Destabilizing walls}

In this section, we discuss some basic properties of destabilizing walls. The destabilizing walls on the $(s, t)$-plane of stability conditions on $D^{b}\left(\mathbb{P}^{2}\right)$ are discussed in [ABCH13, Section 6]. In the $D^{b}$ (qgr- $\left.S\right)$ case, the behavior of the walls is similar to that in the $\mathbb{P}^{2}$ case. As an application, we get a GIT construction of moduli of stable objects in $D^{b}(\operatorname{qgr}-S)$.

The potential wall associated with a pair of invariants $\left(r, c_{1}, \chi\right)$ is given by

$$
W_{r, c_{1}, \chi}^{\mathrm{pot}}:=\bigcup_{\left(r^{\prime}, c_{1}^{\prime}, \chi^{\prime}\right)}\left\{(s, t) \mid \mu_{s, t}\left(r, c_{1}, \chi\right)=\mu_{s, t}\left(r^{\prime}, c_{1}^{\prime}, \chi^{\prime}\right)\right\} .
$$

In the Hilbert scheme case, where $\left(r, c_{1}, \chi\right)=(1,0,1-n)$ (and $\left(r, c_{1}, \chi\right)=(-1,0, n-1)$ when $s \geqslant 0)$, the potential walls form the set

$$
\left\{(s, t) \mid-\frac{c_{1}^{\prime}}{2}\left(s^{2}+t^{2}\right)+\left(\chi^{\prime}-(1-n) r^{\prime}-\frac{3}{2} c_{1}^{\prime}\right) s-n c_{1}^{\prime}=0\right\} .
$$

When $c_{1}^{\prime}=0$, the wall is the $t$-axis. When $c_{1}^{\prime} \neq 0$, these walls are nested semicircles with center $x=\left(\chi^{\prime}-(1-n) r^{\prime}-\frac{3}{2} c_{1}^{\prime}\right) / c_{1}^{\prime}$ and radius $\operatorname{Rad}=\sqrt{x^{2}-2 n}$. In general, we have the following special case of the lemma on nested walls [Mac14, Theorem 3.1].

LEMma 3.1. The potential walls $W_{1,0,1-n}^{\text {pot }}$ in the second quadrant of the $(s, t)$-plane and $W_{-1,0, n-1}^{\text {pot }}$ in the first quadrant are formed by nested semicircles. 


\section{Li And X. ZhaO}

We define the actual wall $W_{r, c_{1}, \chi}^{\text {act }}$ with respect to characters $\left(r, c_{1}, \chi\right)$ as

$$
\left\{(s, t) \mid \exists \text { strictly semistable } \mathcal{F} \text { under }\left(Z_{s, t}, \operatorname{Coh}_{\# s}\right) \text { with invariant }\left(r, c_{1}, \chi\right)\right\} \text {. }
$$

By definition, $W_{r, c_{1}, \chi}^{\text {act }} \subset W_{r, c_{1}, \chi}^{\text {pot }}$. On each quadrant, $W_{1,0,1-n}^{\text {act }}$ is also formed by nested semicircular walls.

Lemma 3.2. For any $k \in \mathbb{Z}$, the sheaf $\mathcal{O}(k)$ (respectively, $\mathcal{O}(k)[1]$ ) is a stable object under stability condition $\left(Z_{s, t}, \mathrm{Coh}_{\# s}\right)$ for $s<k$ (respectively, $s \geqslant k$ ).

This lemma was proved for $\mathbb{P}^{2}$ in [ABCH13]. The general case can be proved similarly.

The group $\widetilde{\mathrm{GL}^{+}(2, \mathbb{R})}$ acts on the space of stability conditions by the $\mathrm{GL}^{+}(2, \mathbb{R})$-action on the central charge and the homological shift on the heart structure. In particular, an element $\phi$ in the subgroup $\mathbb{R}$ acts on $(Z, \mathcal{A})$ as follows: if $\phi$ is an integer, then $\phi \circ(Z, \mathcal{A})=(Z[\phi], \mathcal{A}[\phi])$, where

$$
\mathcal{A}[\phi]=\{A[\phi] \mid A \text { is an object of } \mathcal{A}\} \quad \text { and } Z[i](A):=(-1)^{i} Z(A) .
$$

If $0<\phi<1$, then $\mathcal{A}[\phi]:=\left\langle\mathcal{T}_{\phi}, \mathcal{F}_{\phi}[1]\right\rangle$ and $Z[\phi](E):=e^{-i \pi \phi} Z(E)$ with

$$
\begin{aligned}
& \left.\mathcal{T}_{\phi}=\langle T \in \mathcal{A}| T \text { is stable with } \operatorname{Arg}(Z(T))>\phi \pi\right\rangle, \\
& \left.\mathcal{F}_{\phi}=\langle F \in \mathcal{A}| F \text { is stable with } \operatorname{Arg}(Z(F)) \leqslant \phi \pi\right\rangle .
\end{aligned}
$$

Stable objects remain stable under this $\mathbb{R}$-action.

Proposition 3.3 ([ABCH13, Proposition 7.5]). Let $k$ be an integer. If a pair of real numbers $(s, t)$ satisfies

$$
(s-k)^{2}+t^{2}<1
$$

then there exists $\phi_{s, t, k} \in \mathbb{R}$ (not canonically defined) such that under its action,

$$
\left(Z_{s, t}\left[\phi_{s, t, k}\right], \mathrm{Coh}_{\# s}\left[\phi_{s, t, k}\right]\right)
$$

can be identified with $(Z, \mathcal{A}(k))$ for a suitable choice of central charge $\left(z_{-1}, z_{0}, z_{1}\right)$ for $Z$.

Each semidisc $\left\{(s, t) \mid(s-k)^{2}+t^{2}<1\right\}$ is called a quiver region.

Consider a central charge $\left(z_{-1}, z_{0}, z_{1}\right)$ of $\mathcal{A}(k)$ :

$$
\left(z_{-1}, z_{0}, z_{1}\right)=: \vec{z}=\vec{a}+i \vec{b},
$$

where $\vec{a}$ and $\vec{b}$ are real vectors. Fix three non-negative integers $\left(n_{-1}, n_{0}, n_{1}\right)=\vec{n}$, and define the weight character

$$
\vec{\rho}=-\vec{a}+\vec{b}\left(\frac{\vec{n} \cdot \vec{a}}{\vec{n} \cdot \vec{b}}\right) .
$$

Note that $\vec{n} \cdot \vec{\rho}=0$. An object $E$ in $\mathcal{A}(k)$ with dimension vector $\vec{n}$ is stable (respectively, semistable) with respect to the central charge $\vec{z}$ if and only if for any proper subobject $E^{\prime}$ with dimension vector $\vec{n}^{\prime}$, one has

$$
\left.\vec{n}^{\prime} \cdot \vec{\rho}<0 \quad \text { (respectively, } \vec{n}^{\prime} \cdot \vec{\rho} \leqslant 0\right) .
$$

Remark 3.4. The weight character $\vec{\rho}$ does not change when rescaling $\vec{z}$ by $e^{i \theta}$; in particular, it is invariant under the action of $\phi_{s, t, k}$ in Proposition 3.3. The explicit formula of $\vec{\rho}_{s, t,-k}$ is given as follows. 


\section{MMP FOR DEFORMED HILB $\mathbb{P}^{2}$}

Example 3.5. Consider the dimension vector $\vec{n}=(n, 2 n+1, n)$. When $0 \leqslant k<\sqrt{2 n}$, in the quiver region corresponding to $\mathcal{A}(-k)$, the character $\vec{\rho}_{s, t,-k}$ is given by

$$
\begin{aligned}
& \frac{t s}{t^{2} / 2+2 n-s^{2}}\left((s+k+1)^{2}-\frac{t^{2}}{2},-(s+k)^{2}+\frac{t^{2}}{2},(s+k-1)^{2}-\frac{t^{2}}{2}\right) \\
& \quad+t(s+k+1,-s-k, s+k-1) .
\end{aligned}
$$

In particular, as $t$ tends to 0 , the character $\vec{\rho}_{s, 0+,-k}$ of $G / \mathbb{C}^{\times}$is, up to a scalar, given by

$$
\begin{gathered}
\left(\left(s^{2}+2 n\right)(k+1)+s\left(2 n+(k+1)^{2}\right),-\left(s^{2}+2 n\right) k-s\left(2 n+k^{2}\right),\right. \\
\left.\left(s^{2}+2 n\right)(k-1)+s\left(2 n+(k-1)^{2}\right)\right) .
\end{gathered}
$$

When $s$ decreases from $-k+1$ to $-k-1$, the character $\vec{\rho}_{s, 0+,-k}$ decreases from $\left(1,-n_{-1} / n_{0}, 0\right)$ to $\left(0, n_{1} / n_{0},-1\right)$, up to a positive scalar. In particular, when $s=-k$, as $t$ tends to 0 , up to a positive scalar, $\vec{\rho}_{-k, 0+,-k}$ is $\left(n_{1}, 0,-n_{-1}\right)$. This character corresponds to the destabilizing wall with dimension vector $(0,1,0)$ (in other words, the wall of $\mathcal{O}(-k)[1])$.

Consider the space of characters $\vec{\rho}$; since the subobjects of $E$ have only finitely many possible numerical types, there are finitely many walls in the space of characters $\vec{\rho}$ on which an object $E$ of dimension vector $\vec{n}$ could be strictly semistable with respect to $\vec{\rho}$. These walls divide the space into chambers. When $\vec{\rho}$ varies in a fixed chamber, the moduli space of stable objects remains the same, so one may choose an integral weight character $\vec{\rho}$ as the representative of the chamber. By [Kin94, Proposition 3.1] by King, the moduli space of (semi)stable objects with respect to the central charge $Z$ consists of $\vec{\rho}$-(semi)stable points under the $G$-action.

Define $X$ to be the affine closed subscheme of $\operatorname{Hom}\left(\mathbb{C}^{n_{-1}} \otimes \mathcal{O}(k-1), \mathbb{C}^{n_{0}} \otimes \mathcal{O}(k)\right) \times \operatorname{Hom}\left(\mathbb{C}^{n_{0}} \otimes\right.$ $\left.\mathcal{O}(k), \mathbb{C}^{n_{1}} \otimes \mathcal{O}(k+1)\right)$ consisting of complexes, in other words, the subscheme defined by equations coming from the composition of morphisms being 0 . Let $G$ be the reductive group $\operatorname{GL}\left(n_{-1}, \mathbb{C}\right) \times$ $\operatorname{GL}\left(n_{0}, \mathbb{C}\right) \times \operatorname{GL}\left(n_{1}, \mathbb{C}\right) / \mathbb{C}^{\times}$and $\vec{\rho}$ the character $\left(\operatorname{det}^{\rho_{-1}}\right.$, $\operatorname{det}^{\rho_{0}}$, det $\left.^{\rho_{1}}\right)$ of $G$. This character is well defined since $\vec{\rho} \cdot \vec{n}$ is 0 . When $\vec{n}$ is primitive (that is, $\operatorname{gcd}\left(n_{-1}, n_{0}, n_{1}\right)=1$ ), the group $G$ acts freely on stable points on $X$.

As explained by Ginzburg [Gin12, Chapter 2.2], the moduli space of $Z$-semistable objects can be constructed as a GIT quotient:

$$
\operatorname{Proj} \bigoplus_{n \geqslant 0} \mathbb{C}[X]^{G, \vec{\rho}^{n}}
$$

Notation 3.6. We write $\mathfrak{M}^{\vec{\rho}-s s}(\vec{n}):=X / / \vec{\rho} G=X^{\vec{\rho}-s s} / / G$ for the moduli space of $\vec{\rho}$-semistable objects in $\mathcal{A}(k)$ of dimension vector $\vec{n}$.

Proposition 3.7. (i) Given $n>0$, for any $s<0$ and $t \gg 1$, the moduli space of stable objects with invariants $\left(r, c_{1}, \chi\right)=(1,0,1-n)$ under $\left(Z_{s, t}, \mathrm{Coh}_{\# s}\right)$ is the same as the deformed Hilbert scheme $\operatorname{Hilb}^{n} S$.

(ii) There are only finitely many actual destabilizing walls for $\mathrm{Hilb}^{n} S$.

Proof. Let $\mathcal{I}$ be a torsion-free sheaf with $\left(r, c_{1}, \chi\right)=(1,0,1-n)$. When $k=0$, the object $\mathcal{I}[1]$ lies in $\mathcal{A}(0)$ as an object with dimension vector $\vec{n}=(n, 2 n+1, n)$. By [NS07, Propositions 7.7 and 6.20], if $\vec{\rho}_{m}$ is $((2 n+1)(m-1), n,-(2 n+1) m)$, then for $m \gg 1$, the space $X^{\vec{\rho}_{m}-s s}$ consists of complexes which are quasi-isomorphic to $\mathcal{I}[1]$ for some torsion-free sheaf $\mathcal{I}$ with invariants $(1,0,1-n)$. By the formula in Example 3.5, as $s$ tends to 0 , the character $\vec{\rho}_{s, t}$ is proportional to $\vec{\rho}_{m}$ as $m$ tends to infinity. Therefore, there is an open area $A$ in the region $\left\{(s, t) \mid s^{2}+t^{2}<1, s<0\right\}$ 


\section{Li AND X. ZhaO}

whose boundary contains $(0,0<t<1)$ such that the stable objects with invariants $(1,0,1-n)$ under $\left(Z_{s, t}, \mathrm{Coh}_{\# s}\right)$ are the same as those stable objects in the Gieseker-Mumford sense.

The second statement follows by exactly the same argument as in [ABCH13, Corollary 7.7].

\section{Properties of moduli of Bridgeland-stable objects}

The aim of this section is to show the smoothness and irreducibility of moduli spaces of stable objects of character $(1,0,1-n)$. The smoothness is proved in Section 4.1, and the irreducibility is given in Section 4.3.

\section{1 $\mathrm{Ext}^{2}$ vanishing and smoothness}

The goal of this subsection is to show Theorem 4.3: the moduli space of Bridgeland-stable objects of character $(1,0,1-n)$ is smooth for any generic stability condition $\vec{\rho}$. The difficulty here is the Ext $^{2}$ vanishing for stable objects. For a Gieseker stable sheaf $E$, the shift $E(-3)$ is also stable and of smaller slope, so we have $\operatorname{Ext}^{2}(E, E) \cong \operatorname{Hom}(E, E(-3))^{*}=0$. It is a standard consequence that the moduli of Gieseker stable sheaves is smooth. However, for a stable object $E$ under a Bridgeland-stability condition $\left(Z_{s, t}, \mathrm{Coh}_{\# s}\right)$, the shift $E(-3)$ may be stable not under the same stability condition, but only under $\left(Z_{s-3, t}, \mathrm{Coh}_{\#(s-3)}\right)$. In order to prove a vanishing property of $\operatorname{Ext}^{2}(E, E) \cong \operatorname{Hom}(E, E(-3))^{*}$, we need to develop a method to compare slopes of stable objects under different stability conditions. This is achieved in Lemma 4.2, which is the technical core of this paper.

First, we introduce the following notation.

Notation 4.1. Given a point $(\tilde{s}, \tilde{t})$ on the second quadrant, let $W_{(\tilde{s}, \tilde{t})}$ be the unique semicircle with center at $x$ and radius $\sqrt{x^{2}-2 n}$ that crosses $(\tilde{s}, \tilde{t})$.

Note that as we mentioned in Section 3, such a semicircle does not intersect any other potential walls for the character $(1,0,1-n)$. In particular, if an object of character $(1,0,1-n)$ is stable under $(\tilde{s}, \tilde{t})$, then it is stable under all stability conditions on $W_{(\tilde{s}, \tilde{t})}$.

Lemma 4.2. Let $\mathcal{F}$ be a stable object in $\left(Z_{s_{0}, t_{0}}, \mathrm{Coh}_{\# s_{0}}\right)$ (for some $\left.s_{0}<0\right)$ with numerical class $\left(r, c_{1}, \chi\right)=(1,0,1-n)$. Then we have $\operatorname{Hom}(\mathcal{F}, \mathcal{F}[2])=0$.

Proof. Case I: The semicircle $W_{\left(s_{0}, t_{0}\right)}$ has radius greater than $\frac{3}{2}$. Since the actual destabilizing walls of $\mathcal{F}$ are nested, $\mathcal{F}$ is a stable object under $\left(Z_{s, t}, \operatorname{Coh}_{\# s}\right)$ for all $(s, t) \in W_{\left(s_{0}, t_{0}\right)}$. It follows from the definition of Bridgeland stability that $\mathcal{F}(-3)$ is a stable object under $\left(Z_{s-3, t}, \mathrm{Coh}_{\#(s-3)}\right)$ for any $(s, t) \in W_{\left(s_{0}, t_{0}\right)}$. These points form the semicircle $W_{\left(s_{0}, t_{0}\right)}-(3,0)$. Since the radius of $W_{\left(s_{0}, t_{0}\right)}$ is greater than $\frac{3}{2}$, these two semicircles intersect at a point $\left(s_{1}, t_{1}\right)$. Both $\mathcal{F}$ and $\mathcal{F}(-3)$ are stable under $\left(Z_{s_{1}, t_{1}}, \mathrm{Coh}_{\# s_{1}}\right)$, and we can compare their slopes.

In $\mathrm{Coh}_{\# s_{1}}$, under the central charge $Z_{s_{1}, t_{1}}$, the slope of $\mathcal{F}$ is $\left(-s_{1} / 2+\left(t_{1}^{2}+2 n\right) / 2 s_{1}\right) / t_{1}$, and the slope of $\mathcal{F}(-3)$ is $\left(-\left(s_{1}+3\right) / 2+\left(t_{1}^{2}+2 n\right) /\left(2 s_{1}+6\right)\right) / t_{1}$. Because $s_{1}<-3$, we have

$$
\left(-\frac{s_{1}}{2}+\frac{t_{1}^{2}+2 n}{2 s_{1}}\right) / t_{1}>\left(-\frac{s_{1}+3}{2}+\frac{t_{1}^{2}+2 n}{2 s_{1}+6}\right) / t_{1} .
$$

Thus $\operatorname{Hom}(\mathcal{F}, \mathcal{F}(-3))=0$, and $\operatorname{Hom}(\mathcal{F}, \mathcal{F}[2])=0$ by Serre duality.

Case II: The semicircle $W_{\left(s_{0}, t_{0}\right)}$ has radius less than or equal to $\frac{3}{2}$. Let $k$ be the positive integer such that

$$
(k+1)(k+2) / 2 \leqslant n<(k+2)(k+3) / 2 .
$$




\section{MMP FOR DEFORMED HiLb $\mathbb{P}^{2}$}

We want to show that in this case, both $\mathcal{F}$ and $\mathcal{F}(-3)[1]$ are in the heart $\mathcal{A}(-k-3)$.

To see this, first note that the semicircle $W_{(-k-1,0)}$ has radius at least $\frac{1}{2}$, and by Lemma 5.4 , for stability conditions below this wall, there is no stable object with invariant $(1,0,1-n)$; hence, $W_{\left(s_{0}, t_{0}\right)}$ lies above $W_{(-k-1,0)}$. Also, the radius of $W_{(-k, 0)}$ is greater than $\frac{3}{2}$; hence, $W_{\left(s_{0}, t_{0}\right)}$ lies below $W_{(-k, 0)}$. Therefore, the right edge of $W_{\left(s_{0}, t_{0}\right)}$ falls into the interval $(-k-1,-k)$, which is contained in the quiver region for the heart $\mathcal{A}(-k)$. So a shift of $\mathcal{F}$, say $\mathcal{F}[l]$, is stable in $\mathcal{A}(-k)$. On the other hand, since $(k+1)(k+2) / 2 \leqslant n$, the object $\mathcal{F}$ can be written as the cohomology sheaf of the complex

$$
\mathcal{O}(-k-1)^{\oplus n-k(k-1) / 2} \rightarrow \mathcal{O}(-k)^{\oplus 2 n-k^{2}+1} \rightarrow \mathcal{O}(-k+1)^{\oplus n-k(k+1) / 2}
$$

at the middle term, so $l$ must be 1 . Therefore, $\mathcal{F}[1]$ is an object in $\mathcal{A}(-k)$, and $\mathcal{F}(-3)[1]$ is an object in $\mathcal{A}(-k-3)$.

Also, $W_{\left(s_{0}, t_{0}\right)}$ lies above $W_{(-k-1,0)}$; hence, its left edge is to the left of $-k-2$. By our assumption, its radius is not greater than $\frac{3}{2}$, so its left edge is to the right of $-k-4$. Combining these two observations, the left edge of $W_{\left(s_{0}, t_{0}\right)}$ falls into the quiver region for the heart $\mathcal{A}(-k-3)$. Now, assume that $\mathcal{F}[m]$ is stable in $\mathcal{A}(-k-3)$. As $n<(k+2)(k+3) / 2$, the object $\mathcal{F}$ can be written as the cohomology sheaf of the complex

$$
\mathcal{O}(-k-4)^{\oplus(k+2)(k+3) / 2-n} \rightarrow \mathcal{O}(-k-3)^{\oplus(k+3)^{2}-1-2 n} \rightarrow \mathcal{O}(-k-2)^{\oplus(k+3)(k+4) / 2-n}
$$

at the third term, so $m$ is 0 . Therefore, $\mathcal{F}$ is an object in $\mathcal{A}(-k-3)$.

Now, we have

$$
\operatorname{Hom}(\mathcal{F}, \mathcal{F}(-3))=\operatorname{Hom}(\mathcal{F},(\mathcal{F}(-3)[1])[-1])=0,
$$

where the last equality follows from the fact that both $\mathcal{F}$ and $\mathcal{F}(-3)[1]$ are in the heart $\mathcal{A}(-k-3)$. By Serre duality, $\operatorname{Hom}(\mathcal{F}, \mathcal{F}[2])=0$.

This lemma leads to the smoothness of moduli of stable objects under generic stability conditions. Here, we have a detailed analysis of the GIT construction in our situation, which gives a more concrete proof of the smoothness.

Recall from Section 3 that for a stability condition, by applying the trick of slicing down the wall, we can assume that the stability condition lies in the quiver region for the heart $\mathcal{A}(-k)$, and the moduli of semistable objects is given by the GIT quotient

$$
\mathfrak{M}^{\vec{\rho}-s s}(\vec{n}):=X / / \vec{\rho} G=X^{\vec{\rho}-s s} / / G .
$$

Here, when $n \geqslant(k+1) k / 2$,

$$
\vec{n}=\left(n_{-1}, n_{0}, n_{1}\right)=\left(n-\frac{(k-1) k}{2}, 2 n-k^{2}+1, n-\frac{(k+1) k}{2}\right),
$$

$X$ is the affine closed subscheme of

$$
M=\operatorname{Hom}\left(\mathbb{C}^{n_{-1}} \otimes \mathcal{O}(k-1), \mathbb{C}^{n_{0}} \otimes \mathcal{O}(k)\right) \times \operatorname{Hom}\left(\mathbb{C}^{n_{0}} \otimes \mathcal{O}(k), \mathbb{C}^{n_{1}} \otimes \mathcal{O}(k+1)\right)
$$

consisting of complexes, and $\vec{\rho}$ is the character associated to the stability condition. We denote a point in $M$ by a pair of matrices $(I, J)$, and $(I, J) \in X$ if $J \circ I=0$.

THEOREM 4.3. For a generic $\vec{\rho}$ not on any actual destabilizing wall, the moduli space $\mathfrak{M}^{\vec{\rho}-s}(\vec{n})$ of stable objects is smooth.

Proof. First, observe that the dimension of $M$ is $3 n_{-1} n_{0}+3 n_{0} n_{1}$ and $J \circ I$ has $6 n_{-1} n_{1}$ equations. 


\section{Li And X. ZhaO}

The dimension of the Zariski tangent space of $X$ at a point $\mathbf{K}=\left(I_{0}, J_{0}\right)$ is the dimension of

$$
\operatorname{Hom}_{\mathbb{C}}\left(\mathbb{C}[M] /(J \circ I), \mathbb{C}[t] /\left(t^{2}\right)\right)
$$

at $\left(I_{0}, J_{0}\right)$. Each tangent direction can be written as $\left(I_{0}, J_{0}\right)+t\left(I_{1}, J_{1}\right)$. In order to satisfy that $J \circ I \in\left(t^{2}\right)$, we need

$$
J_{0} \circ I_{1}+J_{1} \circ I_{0}=0 .
$$

Now, consider the space $\operatorname{Hom}^{1}(\mathbf{K}, \mathbf{K})$ consisting of diagrams

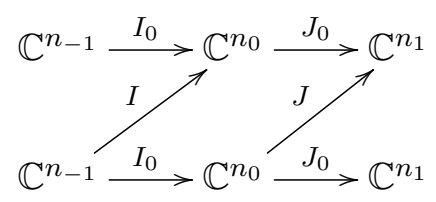

and the space $\operatorname{Hom}^{2}(\mathbf{K}, \mathbf{K})$ consisting of diagrams

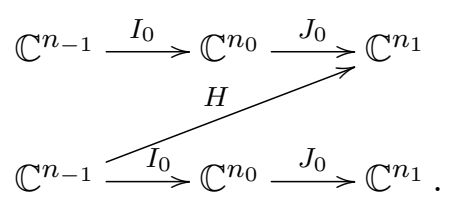

There is a natural map $d^{1}: \operatorname{Hom}^{1}(\mathbf{K}, \mathbf{K}) \rightarrow \operatorname{Hom}^{2}(\mathbf{K}, \mathbf{K})$ obtained by setting $H=J_{0} \circ I+$ $J \circ I_{0}$. Hence, the Zariski tangent space can be identified exactly with the kernel of $d^{1}$. Note that the cokernel of $d^{1}$ is by definition $\operatorname{Ext}^{2}(\mathbf{K}, \mathbf{K})$, which is 0 for stable points $\mathbf{K}$ by Lemma 4.2 . So $d^{1}$ is surjective, and the Zariski tangent space has dimension $\operatorname{dim} \operatorname{Hom}^{1}(\mathbf{K}, \mathbf{K})-\operatorname{dim} \operatorname{Hom}^{2}(\mathbf{K}, \mathbf{K})=$ $3 n_{0}\left(n_{1}+n_{-1}\right)-6 n_{-1} n_{1}$, which is exactly the dimension of $M$ minus the number of equations. Hence, $X^{\rho-s}$ is smooth. Furthermore, since $\left(n_{-1}, n_{0}, n_{1}\right)=\left(n-(k-1) k / 2,2 n-k^{2}+1, n-\right.$ $(k+1) k / 2)$ is primitive and $\vec{\rho}$ is generic, $G$ acts freely on $X^{\rho-s}=X^{\rho-s s}$. By Luna's étale slice theorem, $X^{\rho-s} \rightarrow X^{\rho-s} / / G$ is a principal bundle. Since $X^{\rho-s}$ is smooth, by [GD67, Proposition 17.7.7], the quotient space is also smooth.

Later, in order to prove the irreducibility result, we will need a stronger version of Lemma 4.2. Recall that on each wall, all S-equivalent semistable objects (that is, objects whose stable factors are the same up to rearrangement) are contracted to one point. Let $\mathcal{F}$ be a strictly semistable object at $\left(s_{0}, t_{0}\right)$ with invariants $(1,0,1-n)$. Then $\mathcal{F}$ has a filtration in $\mathrm{Coh}_{\# s_{0}}$

$$
\mathcal{F}=\mathcal{F}_{m} \supset \mathcal{F}_{m-1} \supset \cdots \supset \mathcal{F}_{1} \supset \mathcal{F}_{0}=0
$$

such that each factor $\mathcal{E}_{i}:=\mathcal{F}_{i} / \mathcal{F}_{i-1}$ is stable under $Z_{s_{0}, t_{0}}$. We call this the Jordan-Hölder filtration, and each $\mathcal{E}_{i}$ is called a stable factor of $\mathcal{F}$ under stability condition $\left(s_{0}, t_{0}\right)$. For any point $(s, t)$ on $W_{\left(s_{0}, t_{0}\right)}$, it is a standard consequence of Lemma 3.1 that

$$
\mu_{s, t}\left(\mathcal{E}_{i}\right)=\mu_{s, t}(\mathcal{F}) .
$$

To prove the irreducibility result, we need to control Ext ${ }^{2}$ between stable factors of strictly semistable objects.

Lemma 4.4. Let $\mathcal{E}_{1}, \ldots, \mathcal{E}_{m}$ be the stable factors of $\mathcal{F}$ as above; then we have

$$
\operatorname{Hom}\left(\mathcal{E}_{i}, \mathcal{E}_{j}[2]\right)=0
$$

for all $1 \leqslant i, j \leqslant m$. In particular, for any semistable subobject $\mathcal{E}$ of $\mathcal{F}$ in $\mathrm{Coh}_{\# s_{0}}$ with the same $\sigma_{s_{0}, t_{0}}$-slope as that of $F$, we have $\operatorname{Hom}(\mathcal{F} / \mathcal{E}, \mathcal{E}[2])=0$. 


\section{MMP FOR DEFORMED HiLb $\mathbb{P}^{2}$}

Proof. In order to apply the same trick as in Lemma 4.2, we first show that each $\mathcal{E}_{i}$ is stable on the whole $W_{\left(s_{0}, t_{0}\right)}$. Since $\mathcal{E}_{i}$ is a stable factor, the wall $W_{\left(s_{0}, t_{0}\right)}$ is also a numerical wall for $\mathcal{E}_{i}$. If $\mathcal{E}_{i}$ leaves the category $\mathrm{Coh}_{\# s}$ or becomes unstable, two numerical walls for $\mathcal{E}_{i}$ intersect. But this contradicts the nested walls lemma [Mac14, Theorem 3.1].

Now, we can prove the statement as in the proof of Lemma 4.2. When the semicircle $W_{\left(s_{0}, t_{0}\right)}$ has radius greater than $\frac{3}{2}$, we have $\mu_{s, t}\left(\mathcal{E}_{i}(-3)\right)=\mu_{s, t}\left((\mathcal{F}(-3))<\mu_{s, t}(F)=\mu_{s, t}\left(\mathcal{E}_{j}\right)\right.$ on $W_{\left(s_{0}, t_{0}\right)}$. When the radius is not greater than $\frac{3}{2}$, the objects $\mathcal{E}_{j}$ and $\mathcal{E}_{i}(-3)[1]$ are both in $\mathcal{A}(-k-3)$ (since $\mathcal{F}(-3)[1]$ is in $\mathcal{A}(-k-3)$ and $\mathcal{E}_{i}(-3)$ has the same slope of $\mathcal{F}(-3)$ along the wall). In either case, $\operatorname{Hom}\left(\mathcal{E}_{j}, \mathcal{E}_{i}(-3)\right)=0$. So we get

$$
\operatorname{Hom}\left(\mathcal{E}_{i}, \mathcal{E}_{j}[2]\right) \cong \operatorname{Hom}\left(\mathcal{E}_{j}, \mathcal{E}_{i}(-3)\right)^{*}=0 .
$$

This concludes the proof.

\subsection{Dimension estimate and extensions}

In this subsection, we study the properties of objects coming from extensions. All through this subsection, we assume that stability conditions are in the quiver region for the heart $\mathcal{A}(-k)$ for $k \geqslant 1$. The main technical result is Lemma 4.7, which will be used in Section 4.3 for the proof of irreducibility.

We have the dimension vector $\vec{n}$ and the character $\vec{\rho}$ as before. For any dimension vector $\vec{m}$, we define $X(\vec{n})$ to be the affine closed subscheme of Hom $\left(\mathbb{C}^{n_{-1}} \otimes \mathcal{O}(k-1), \mathbb{C}^{n_{0}} \otimes \mathcal{O}(k)\right) \times$ $\operatorname{Hom}\left(\mathbb{C}^{n_{0}} \otimes \mathcal{O}(k), \mathbb{C}^{n_{1}} \otimes \mathcal{O}(k+1)\right)$ consisting of complexes in $\mathcal{A}(-k)$. Let $G_{\vec{n}}$ be the reductive group $\mathrm{GL}\left(n_{-1}, \mathbb{C}\right) \times \mathrm{GL}\left(n_{0}, \mathbb{C}\right) \times \mathrm{GL}\left(n_{1}, \mathbb{C}\right) / \mathbb{C}^{\times}$that acts on $X(\vec{n})$, and let $\vec{\rho}$ be the character $\left(\operatorname{det}^{\rho_{-1}}, \operatorname{det}^{\rho_{0}}, \operatorname{det}^{\rho_{1}}\right)$ of $G$. The character is well defined when $\vec{\rho} \cdot \vec{n}$ is 0 . We will use $X^{\vec{\rho}-s s}(\vec{n}) \subset$ $X(\vec{n})$ to denote the space of $\vec{\rho}$-semistable objects in $\mathcal{A}(-k)$ of dimension vector $\vec{n}$.

Definition 4.5. Suppose $\vec{n}=\vec{n}^{\prime}+\vec{n}^{\prime \prime}$ with $\vec{n}^{\prime} \cdot \vec{\rho}=\vec{n}^{\prime \prime} \cdot \vec{\rho}=0$. Choose $\mathbf{F} \in X^{\vec{\rho}-s s}\left(\vec{n}^{\prime}\right)$ and $\mathbf{G} \in X^{\vec{\rho}-s s}\left(\vec{n}^{\prime \prime}\right)$. We write $X(\mathbf{F}, \mathbf{G})$ for the subspace in $X(\vec{n})$ consisting of objects on the $G_{\vec{n}^{-}}$ orbits of $\mathbf{K}$ that can be written as an extension of $\mathbf{G}$ by $\mathbf{F}$ :

$$
0 \rightarrow \mathbf{F} \rightarrow \mathbf{K} \rightarrow \mathbf{G} \rightarrow 0 \text {. }
$$

We also write $X\left(\vec{n}^{\prime}, \vec{n}^{\prime \prime}\right)$ for the union of all $X(\mathbf{F}, \mathbf{G})$.

We have the following dimension estimate.

Lemma 4.6. Suppose that the dimension vector $\vec{n}$ of $\mathbf{K}$ is associated with the numerical class $(1,0,1-n)$ of the Hilbert scheme; then

$$
\operatorname{dim} X(\mathbf{F}, \mathbf{G}) \leqslant-\chi(\mathbf{G}, \mathbf{F})+\operatorname{dim} G_{\vec{n}}-\operatorname{hom}(\mathbf{F}, \mathbf{F})-\operatorname{hom}(\mathbf{G}, \mathbf{G}) .
$$

Proof. Let $\bar{X}(\mathbf{F}, \mathbf{G})$ be the subset of $X(\mathbf{F}, \mathbf{G})$ consisting of objects of the form

$$
I=\left(\begin{array}{cc}
I_{\mathbf{F}} & I(\mathbf{F}, \mathbf{G}) \\
0 & I_{\mathbf{G}}
\end{array}\right), \quad J=\left(\begin{array}{cc}
J_{\mathbf{F}} & J(\mathbf{F}, \mathbf{G}) \\
0 & J_{\mathbf{G}}
\end{array}\right)
$$

for some pair $(I(\mathbf{F}, \mathbf{G}), J(\mathbf{F}, \mathbf{G}))$. The morphisms are shown in the following diagram:

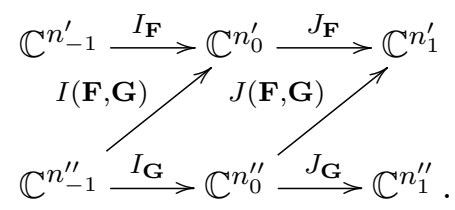




\section{Li AND X. ZhaO}

The pair $(I(\mathbf{F}, \mathbf{G}), J(\mathbf{F}, \mathbf{G}))$ is contained in the kernel of the morphism

$$
d^{1}(\mathbf{G}, \mathbf{F}): \operatorname{Hom}^{1}(\mathbf{G}, \mathbf{F}) \rightarrow \operatorname{Hom}^{2}(\mathbf{G}, \mathbf{F}) .
$$

By Lemma 4.4, we have coker $d^{1}=\operatorname{Ext}^{2}(\mathbf{G}, \mathbf{F})=0$, so $d^{1}(\mathbf{G}, \mathbf{F})$ is surjective, and

$$
\operatorname{dim} \bar{X}(\mathbf{F}, \mathbf{G})=\operatorname{hom}^{1}(\mathbf{G}, \mathbf{F})-\operatorname{hom}^{2}(\mathbf{G}, \mathbf{F}) .
$$

Each element $g \in G_{\vec{n}}$ can be written as a block matrix $(\underset{C}{A} \underset{D}{B})$, where $A \in \operatorname{Hom}^{0}(\mathbf{F}, \mathbf{F})$, $B \in \operatorname{Hom}^{0}(\mathbf{G}, \mathbf{F}), C \in \operatorname{Hom}^{0}(\mathbf{F}, \mathbf{G})$ and $D \in \operatorname{Hom}^{0}(\mathbf{G}, \mathbf{G})$. Note that when $A \in \operatorname{Hom}(\mathbf{F}, \mathbf{F})$, $D \in \operatorname{Hom}(\mathbf{G}, \mathbf{G})$ and $C=0$, we have $g \bar{X}(\mathbf{F}, \mathbf{G}) \subset \bar{X}(\mathbf{F}, \mathbf{G})$. Therefore,

$$
\begin{aligned}
\operatorname{dim} X(\mathbf{F}, \mathbf{G}) & =\operatorname{dim}\left(G_{\vec{n}} \bar{X}(\mathbf{F}, \mathbf{G})\right) \\
& \leqslant \operatorname{dim} G_{\vec{n}}+\operatorname{dim} \bar{X}(\mathbf{F}, \mathbf{G})-\operatorname{hom}^{0}(\mathbf{G}, \mathbf{F})-\operatorname{hom}(\mathbf{F}, \mathbf{F})-\operatorname{hom}(\mathbf{G}, \mathbf{G}) \\
& =\operatorname{dim} G_{\vec{n}}-\chi(\mathbf{G}, \mathbf{F})-\operatorname{hom}(\mathbf{F}, \mathbf{F})-\operatorname{hom}(\mathbf{G}, \mathbf{G}) .
\end{aligned}
$$

This finishes the claim.

LEMMA 4.7. Let $\vec{n}$ be the dimension vector of a destabilizing factor, and let $\vec{\rho}$ be a character corresponding to a generic stability condition for $\vec{n}$. Assume that $X^{\vec{\rho}-s s}(\vec{n})$ is non-empty; then each irreducible component of $X^{\vec{\rho}-s s}(\vec{n})$ is of dimension $-\chi(\vec{n}, \vec{n})+\operatorname{dim} G_{\vec{n}}$.

Proof. The variety $X(\vec{n})$ is the zero locus of $6 n_{-1} n_{1}$ equations in an affine space. It is easy to see that each irreducible component of $X(\vec{n})$ is of dimension at least $-\chi(\vec{n}, \vec{n})+\operatorname{dim} G_{\vec{n}}$.

On the other hand, similarly to the argument in Theorem 4.3 , for any $\rho$-semistable object $\mathbf{F} \in$ $X^{\vec{\rho}-s s}(\vec{n})$, the map $d^{1}: \operatorname{Hom}^{1}(\mathbf{F}, \mathbf{F}) \rightarrow \operatorname{Hom}^{2}(\mathbf{F}, \mathbf{F})$ is surjective by Lemma 4.4 , and the Zariski tangent space at $\mathbf{F}$ is of dimension $-\chi(\vec{n}, \vec{n})+\operatorname{dim} G_{\vec{n}}$. Since $X^{\vec{\rho}-s s}(\vec{n})$ is open in $X(\vec{n})$, we see that each irreducible component of $X^{\vec{\rho}-s s}(\vec{n})$ must be of dimension exactly $-\chi(\vec{n}, \vec{n})+\operatorname{dim} G_{\vec{n}}$.

\subsection{Irreducibility of the moduli spaces}

Now, we want to show the irreducibility of moduli of Bridgeland-stable objects (Theorem 4.11). This is known in the Gieseker chamber, and the way we prove it for other stability conditions is by studying the behavior of moduli of stable objects when the stability condition varies across a wall in a quiver region. Based on the results and methods in Section 4.2, we are able to estimate the dimension of the space of new stable objects after a wall crossing. When the wall is to the left of the vertical wall, we show that after wall crossing, the new stable objects arising as extensions have codimension at least 3 (Lemma 4.10). This will imply that the moduli of stable objects always remain irreducible.

In this subsection, we always assume $\vec{n}$ to be the dimension vector associated with the numerical class $(1,0,1-n)$. Consider an actual wall whose right edge falls in the quiver region for $\mathcal{A}(k)$. Assume that the character $\vec{\rho}$ corresponds to a stability condition on this wall and in this quiver region. Let $\vec{\rho}_{-}$and $\vec{\rho}_{+}$be the characters corresponding, respectively, to stability conditions to the left and right of the given stability condition. In order to control the dimension of new stable objects, we need the following lemma.

Lemma 4.8. Suppose that $\mathbf{K}$ is an object in $X^{\vec{\rho}_{-}-s}(\vec{n}) \backslash X^{\vec{\rho}_{+}-s}(\vec{n})$; then it can be written as a non-trivial extension

$$
0 \rightarrow \mathbf{K}^{\prime} \rightarrow \mathbf{K} \rightarrow \mathbf{K}^{\prime \prime} \rightarrow 0
$$

of objects in $\mathcal{A}(k)$ such that the dimension vector $\vec{n}^{\prime}$ of $\mathbf{K}^{\prime}$ satisfies $\vec{\rho}_{-} \cdot \vec{n}^{\prime}<0=\vec{\rho} \cdot \vec{n}^{\prime}$, and $\operatorname{Hom}\left(\mathbf{K}^{\prime \prime}, \mathbf{K}^{\prime}\right)=0$. Moreover, we have $\chi\left(\mathbf{K}^{\prime \prime}, \mathbf{K}^{\prime}\right)<0$. 


\section{MMP FOR DEFORMED HILB $\mathbb{P}^{2}$}

Proof. By the assumption on $\mathbf{K}$, it is a strictly $\vec{\rho}$-semistable object and is destabilized by a nonzero $\vec{\rho}$-stable subobject $\mathbf{K}^{\prime}$ with $\vec{\rho} \cdot \vec{n}^{\prime}=0$. As $\mathbf{K}$ is $\vec{\rho}_{-}$-stable, we have $\vec{\rho}_{-} \cdot \vec{n}^{\prime}<0$. Denote the quotient by $\mathbf{K}^{\prime \prime}$. Suppose that there is a non-zero element in $\operatorname{Hom}\left(\mathbf{K}^{\prime \prime}, \mathbf{K}^{\prime}\right)$; then its image $\tilde{\mathbf{K}}$ in $\mathbf{K}^{\prime}$ is both a subobject and quotient object of $\mathbf{K}$ in $\mathcal{A}(k)$. Let $\tilde{n}$ be the dimension vector of $\tilde{\mathbf{K}}$; since $\mathbf{K}$ is $\vec{\rho}_{-}$-stable, we get $\vec{\rho}_{-} \cdot \tilde{n}<0<\vec{\rho}_{-} \cdot \tilde{n}$, which gives a contradiction. So $\operatorname{Hom}\left(\mathbf{K}^{\prime \prime}, \mathbf{K}^{\prime}\right)=0$.

The last statement $\chi\left(\mathbf{K}^{\prime \prime}, \mathbf{K}^{\prime}\right)<0$ follows from the existence of non-trivial extensions and Lemma 4.4.

Notation 4.9. We introduce the following notation for Lemma 4.10.

$$
\begin{aligned}
X^{\vec{\rho}-s s}\left(\vec{n}^{\prime}\right)_{c} & :=\left\{\mathbf{K} \in X^{\vec{\rho}-s s}\left(\vec{n}^{\prime}\right) \mid \operatorname{hom}(\mathbf{K}, \mathbf{K})=c\right\} \\
X^{\vec{\rho}-s s}\left(\vec{n}^{\prime}, \vec{n}^{\prime \prime}\right)_{c, d} & :=\left\{\mathbf{K} \in X^{\vec{\rho}-s s}(\mathbf{F}, \mathbf{G}) \mid \operatorname{hom}(\mathbf{F}, \mathbf{F})=c, \operatorname{hom}(\mathbf{G}, \mathbf{G})=d\right\} .
\end{aligned}
$$

LEMma 4.10. The dimension of the space $X^{\vec{\rho}_{-}-s}(\vec{n}) \backslash X^{\vec{\rho}_{+}-s}(\vec{n})$ is at most $-\chi(\vec{n}, \vec{n})+\operatorname{dim} G_{\vec{n}}-3$. Proof. By Lemma 4.8, the space $X^{\vec{\rho}_{-}-s}(\vec{n}) \backslash X^{\vec{\rho}_{+}-s}(\vec{n})$ can be covered by the following pieces:

$$
X^{\vec{\rho}_{-}-s}(\vec{n}) \backslash X^{\vec{\rho}_{+}-s}(\vec{n}) \subset \bigcup_{\vec{m}} X(\vec{m},(\vec{n}-\vec{m})),
$$

where $\vec{m}$ satisfies the following two requirements:

$$
\begin{aligned}
\vec{\rho}_{-} \cdot \vec{m} & <0=\vec{\rho}_{0} \cdot \vec{m}, \\
\chi(\vec{n}-\vec{m}, \vec{m}) & \leqslant 0 .
\end{aligned}
$$

A subtle point is that for objects $\mathbf{K}^{\prime}$ with dimension vector $\vec{m}$, the dimension of $\operatorname{Hom}\left(\mathbf{K}^{\prime}, \mathbf{K}^{\prime}\right)$ may not be a constant. However, we see that this does not affect our dimension estimate. Let $X(\vec{m}, \vec{n}-\vec{m})_{c, d}$ be the space of objects arising as extensions of $\vec{\rho}_{-}$-semistable objects $\mathbf{K}^{\prime}$ and $\mathbf{K}^{\prime \prime}$, where $\mathbf{K}^{\prime}$ has dimension vector $\vec{m}, \operatorname{dim} \operatorname{Hom}\left(\mathbf{K}^{\prime}, \mathbf{K}^{\prime}\right)=c$, the object $\mathbf{K}^{\prime \prime}$ has dimension vector $\left(n_{1}, n_{2}, n_{3}\right)-\vec{m}$, and $\operatorname{dim} \operatorname{Hom}\left(\mathbf{K}^{\prime \prime}, \mathbf{K}^{\prime \prime}\right)=d$.

Note that by Definition 4.5 , for $\mathbf{F}, \mathbf{F}^{\prime} \in X^{\vec{\rho}--s s}(\vec{m})$ and $\mathbf{G}, \mathbf{G}^{\prime} \in X^{\vec{\rho}-s s}(\vec{n}-\vec{m})$, the space $X(\mathbf{F}, \mathbf{G})$ equals $X\left(\mathbf{F}^{\prime}, \mathbf{G}^{\prime}\right)$ when $\mathbf{F}, \mathbf{F}^{\prime}$ are in the same $G_{\vec{m}^{-}}$orbit and $\mathbf{G}, \mathbf{G}^{\prime}$ are in the same $G_{\vec{n}-\vec{m}}$-orbit. By Lemma 4.6, we have

$$
\begin{aligned}
\operatorname{dim} X(\vec{m}, \vec{n}-\vec{m})_{c, d} \leqslant & -\chi(\vec{n}-\vec{m}, \vec{m})+\operatorname{dim} G_{\vec{n}}-c-d+\operatorname{dim}\left(X^{\vec{\rho}_{-}-s s}(\vec{m})_{c} / / G_{\vec{m}}\right) \\
& +\operatorname{dim}\left(X^{\vec{\rho}--s s}(\vec{n}-\vec{m})_{d} / / G_{\vec{n}-\vec{m}}\right) \\
= & -\chi(\vec{n}-\vec{m}, \vec{m})+\operatorname{dim} G_{\vec{n}}-c-d-\left(\operatorname{dim} G_{\vec{m}}-c\right)-\left(\operatorname{dim} G_{\vec{n}-\vec{m}}-d\right) \\
& +\operatorname{dim} X^{\vec{\rho}--s s}(\vec{m})_{c}+\operatorname{dim} X^{\vec{\rho}--s s}(\vec{n}-\vec{m})_{d} \\
\leqslant & -\chi(\vec{n}-\vec{m}, \vec{m})+\operatorname{dim} G_{\vec{n}}-\chi(\vec{n}-\vec{m}, \vec{n}-\vec{m})-\chi(\vec{m}, \vec{m}) \quad \text { by Lemma } 4.7 \\
= & -\chi(\vec{n}, \vec{n})+\operatorname{dim} G_{\vec{n}}+\chi(\vec{m}, \vec{n}-\vec{m}) .
\end{aligned}
$$

Write $\vec{m}=\left(m_{-1}, m_{0}, m_{1}\right)$; then by our assumption on $\vec{m}$, we have

$$
\begin{aligned}
\chi(\vec{m}, \vec{n}-\vec{m}) \leqslant & -\chi(\vec{n}-\vec{m}, \vec{m})+\chi(\vec{m}, \vec{n}-\vec{m}) \\
= & -\chi(\vec{n}, \vec{m})+\chi(\vec{m}, \vec{n}) \\
= & -\left(\vec{n} \cdot \vec{m}-3\left(n_{-1} m_{0}+n_{0} m_{1}\right)+6 n_{-1} m_{1}\right) \\
& +\left(\vec{n} \cdot \vec{m}-3\left(m_{-1} n_{0}+m_{0} n_{1}\right)+6 m_{-1} n_{1}\right) \\
= & -\left(6 n_{-1}-3 n_{0}\right) m_{1}-\left(3 n_{1}-3 n_{-1}\right) m_{0}-\left(3 n_{0}-6 n_{1}\right) m_{-1} \\
= & -3 \vec{m} \cdot(k+1,-k, k-1) .
\end{aligned}
$$




\section{Li AND X. ZhaO}

By the formula below Example 3.5, when s decreases from $-k+1$ to $-k-1$, the character $\vec{\rho}_{s, 0+,-k}$ decreases from $\left(n_{0},-n_{-1}, 0\right)$ to $\left(0, n_{1},-n_{0}\right)$ up to a positive scalar. Therefore, $\vec{\rho}_{-}=\vec{\rho}_{0}+$ $\epsilon\left(0, n_{1},-n_{0}\right)$ for $\epsilon>0$, as well as $\vec{\rho}_{0}=\vec{\rho}_{-}+\epsilon^{\prime}\left(n_{0},-n_{-1}, 0\right)$ for $\epsilon^{\prime}>0$. Since $\vec{n} \cdot(k+1,-k, k-1)=0$ and $\vec{n}^{\perp}$ is spanned by $\left(n_{0},-n_{-1}, 0\right)$ and $\left(0, n_{1},-n_{0}\right)$, the weight character satisfies

$$
\begin{aligned}
(k+1,-k, k-1) & =\frac{k+1}{n_{0}}\left(n_{0},-n_{-1}, 0\right)-\frac{k-1}{n_{0}}\left(0, n_{1},-n_{0}\right) \\
& =\frac{k+1}{n_{0} \epsilon^{\prime}}\left(\vec{\rho}_{0}-\vec{\rho}_{-}\right)-\frac{k-1}{n_{0} \epsilon}\left(\vec{\rho}_{-}-\vec{\rho}_{0}\right) \\
& =a \vec{\rho}_{0}-b \vec{\rho}_{-} .
\end{aligned}
$$

Note that for $k \geqslant 1$ (the quiver region $\mathcal{A}(-k)$ is to the left of the vertical wall), we have $a, b>0$. Hence, $\vec{m} \cdot(k+1,-k, k-1) \geqslant 1$. So

$$
\operatorname{dim}\left(X^{\vec{\rho}_{-}-s}(\vec{n}) \backslash X^{\vec{\rho}_{+}-s}(\vec{n})\right) \leqslant-\chi(\vec{n}, \vec{n})+\operatorname{dim} G_{\vec{n}}-3 .
$$

This concludes the proof of the claim.

Now, we are ready to prove our main theorem on irreducibility.

THEOREM 4.11. For a generic stability condition not on any actual destabilizing wall, the moduli space of stable objects of numerical class $(1,0,1-n)$ is irreducible.

Proof. Again, we use the trick of slicing down the wall to reduce to wall crossing in quiver regions. In the Gieseker chamber, $X^{\vec{\rho}-s}(\vec{n})$ is irreducible since the quotient space, which is exactly the Hilbert scheme of points, is smooth and irreducible. By Propositions 4.7 and 4.10, while going across an actual wall, the dimension of the new stable locus $X^{\vec{\rho}_{-}-s}(\vec{n}) \backslash X^{\vec{\rho}_{-}-s}(\vec{n})$ is smaller than the dimension of any irreducible component of $X^{\vec{\rho}_{--s}}(\vec{n})$ (by at least 3 ). Hence, the new stable locus is contained in the same irreducible component of $X^{\vec{\rho}+-s}(\vec{n})$. So $X^{\vec{\rho}_{-}-s}(\vec{n})$ remains irreducible and the moduli space as the quotient space is also irreducible.

\section{MMP via variation of GIT}

The aim of this section is to show that wall crossing in the space of Bridgeland-stability conditions induces the minimal model program for deformations of Hilbert schemes of points on $\mathbb{P}^{2}$. In Section 5.1, we rephrase some results from variation of GIT in our setup. In Section 5.2, we study the wall-crossing behavior on the second quadrant and show that this completes the theory for $\mathbb{P}^{2}$. For the deformations, wall crossing over the vertical wall is more involved and induces a different picture of MMP. This is studied in detail in Section 5.3.

\subsection{Properties of GIT}

Birational geometry via GIT has been studied in [DH98] by Dolgachev and Hu, and in [Tha96] by Thaddeus. Since we are mainly working with the GIT quotients of affine schemes, we need to rephrase some theorems in our setup. In this section, we recollect some properties in the language of affine GIT.

Let $X$ be an affine algebraic $G$-variety, where $G$ is a reductive group and acts on $X$ via a linear representation. Given a character $\rho: G \rightarrow \mathbb{C}^{\times}$, the stable and semistable loci are written as $X^{\rho-s}$ and $X^{\rho-s s}$, respectively. We write $C[B]^{G, \chi}$ for the $\chi$-semi-invariant functions on $B \subset X$; that is, one has

$$
f\left(g^{-1}(x)\right)=\chi(g) \cdot f(x) \quad \forall g \in G, x \in B
$$




\section{MMP FOR DEFORMED HILB $\mathbb{P}^{2}$}

We denote the GIT quotient by $X / \|_{\rho} G:=\operatorname{Proj} \bigoplus_{n \geqslant 0} \mathbb{C}[X]^{G, \rho^{n}}$ and the morphism from $X^{\rho-s s}$ to $X /{ }_{\rho} G$ by $F_{\rho}$.

In addition, we make the following assumptions on $X$ and $G$ :

(1) There are only finitely many walls in the space of characters on which there are strictly semistable points. In a chamber between two walls, we always have $X^{\rho-s}=X^{\rho-s s}$.

(2) The stable locus $X^{\rho-s}$ is smooth, and the action of $G$ on $X^{\rho-s}$ is free.

(3) The GIT quotient $X / / \rho G$ is projective and connected.

(4) For any characters $\rho$ and $\rho^{\prime}$ such that $X^{\rho-s}$ and $X^{\rho^{\prime}-s}$ are non-empty, the closures of $X^{\rho-s}$ and $X^{\rho^{\prime}-s}$ are in the same irreducible component of $X$.

(5) Given any point $x \in X$, the set of characters $\left\{\rho \mid x \in X^{\rho-s s}\right\}$ is closed.

Definition 5.1. Let $\rho$ be a generic character (that is, not on walls) such that $X^{\rho-s}$ is nonempty; then by our assumptions we have a $G$-principal bundle $X^{\rho-s} \rightarrow X / / \rho=X^{\rho-s} / / G$. For any character $\rho_{0}$ of $G$, let $\mathcal{L}_{\rho, \rho_{0}}$ be the line bundle over $X \|_{\rho} G$ given by the composition of transition functions of the $G$-principal bundle with $\rho_{0}$. In other words, consider the trivial line bundle over $X$ with $G$-action given by the character $\rho_{0}$; then this $G$-line bundle descends to $\mathcal{L}_{\rho, \rho_{0}}$ on $X / \|_{\rho} G$.

Now, we are ready to list some properties from the variation of geometric invariant theory (VGIT).

Proposition 5.2. Let $X$ be an affine algebraic $G$-variety that satisfies Assumptions (1) to (5), and let $\mathcal{L}_{\rho, \rho_{0}}$ be the line bundle defined above.

(i) $\Gamma\left(X / / \rho, \mathcal{L}_{\rho, \rho_{0}}^{\otimes n}\right) \simeq \mathbb{C}\left[X^{\rho-s}\right]^{G, \rho_{0}^{n}}$.

(ii) If $\rho_{+}$and $\rho$ are in the same chamber, then $\mathbb{C}\left[X^{\rho-s}\right]^{G, \rho_{+}^{n}}=\mathbb{C}[X]^{G, \rho_{+}^{n}}$ for $n \gg 1$ and $\mathcal{L}_{\rho, \rho_{+}}$is ample; if $\rho_{0}$ is a generic point on the wall bounding the chamber of $\rho$, then $\mathcal{L}_{\rho, \rho_{0}}$ is nef and semiample.

(iii) Let $\rho_{+}$and $\rho_{0}$ be in the chamber of $\rho$ and on the wall, respectively; then there is an inclusion $X^{\rho_{+}-s s} \subset X^{\rho_{0}-s s}$, which induces a canonical projective morphism $\mathrm{pr}_{+}: X / / \rho_{+} G \rightarrow X / / \rho_{0} G$.

(iv) $A$ (projective, smooth, connected) curve $C$ in $X / /_{\rho_{+}} G$ is contracted by $\mathrm{pr}_{+}$if and only if it is contracted by $X / /_{\rho_{+}} G \rightarrow \operatorname{Proj} \oplus_{n \geqslant 0} \Gamma\left(X / \rho_{\rho_{+}} G, \mathcal{L}_{\rho_{+}, \rho_{0}}^{\otimes n}\right)$.

(v) Let $\rho_{+}$and $\rho_{-}$be in the two chambers on different sides of a wall, and let $\rho_{0}$ be a generic point on the wall. Assume that $X^{\rho_{ \pm}-s}$ are both non-empty; then the morphisms $X /{ }_{\rho_{ \pm}} G \rightarrow X / \rho_{\rho_{0}} G$ are both proper and birational. Moreover, if they are both small, then the rational map $X / \rho_{\rho_{-}} G \rightarrow X / \rho_{\rho_{+}} G$ is a flip with respect to the line bundle $\mathcal{L}_{\rho_{+}, \rho_{0}}$.

Proof. (i) This is true in general for $G$-principal bundles by flat descent theorem; see [Del77, Exposé I, Théorème 4.5].

(ii) and (iii) By Assumption (5), we have $X^{\rho-s} \subset X^{\rho_{*}-s s}$, where $*$ stands for 0 or + . By Assumption (4), the natural map $\mathbb{C}[X]^{G, \rho_{*}^{n}} \rightarrow \mathbb{C}\left[X^{\rho-s}\right]^{G, \rho_{*}^{n}} \simeq \Gamma\left(X / / \rho G, \mathcal{L}_{\rho, \rho_{*}}^{\otimes n}\right)$ is injective for $n \in \mathbb{Z}_{\geqslant 0}$. Hence, the base locus of $\mathcal{L}_{\rho, \rho_{*}}$ is empty. The graded $\operatorname{ring} \mathcal{R}\left(X \|_{\rho} G, \mathcal{L}_{\rho, \rho_{*}}\right) \simeq$ $\bigoplus_{n \geqslant 0} \mathbb{C}\left[X^{\rho-s}\right]^{G, \rho_{*}^{n}}$ is finitely generated over $\mathbb{C}$. When $X^{\rho_{*}-s}$ is non-empty, the canonical morphism $X / / \rho \rightarrow \operatorname{Proj} \bigoplus_{n \geqslant 0} \mathbb{C}\left[X^{\rho-s}\right]^{G, \rho_{*}^{n}}$ is birational and projective. Now, we have morphisms

$$
\operatorname{pr}_{+}: X \|_{\rho} G \rightarrow \operatorname{Proj} \bigoplus_{n \geqslant 0} \mathbb{C}\left[X^{\rho-s}\right]^{G, \rho_{*}^{n}} \rightarrow \operatorname{Proj} \bigoplus_{n \geqslant 0} \mathbb{C}[X]^{G, \rho_{*}^{n}}=X / / \rho_{*} G .
$$




\section{Li AND X. ZhaO}

The morphism $\mathrm{pr}_{+}$maps each $S$-equivariant class with respect to the stability condition $\rho_{*}$ to itself set-theoretically. When $\rho_{+}$is in the same chamber as $\rho$, by Assumption (2), this is an isomorphism, which implies that $\mathcal{L}_{\rho, \rho_{+}}$must be ample and $\mathbb{C}\left[X^{\rho-s}\right]^{G, \rho_{+}^{n}}=\mathbb{C}[X]^{G, \rho_{+}^{n}}$ for $n$ large enough. By the definition of $\mathcal{L}_{\rho, \rho_{+}}$, it extends to a linear map from the space of real characters of $G$ to $\operatorname{NS}_{\mathbb{R}}(X / / \rho)$. Since all elements in the chamber of $\rho$ are mapped into the ample cone, $\mathcal{L}_{\rho, \rho_{0}}$ as the limit must be nef.

(iv) ' $\Leftarrow$ ': by Assumption (4), the map Proj $\oplus_{n \geqslant 0} \mathbb{C}\left[X^{\rho_{+}-s}\right]^{G, \rho_{0}^{n}} \rightarrow \operatorname{Proj} \oplus_{n \geqslant 0} \mathbb{C}[X]^{G, \rho_{0}^{n}}$ is always surjective. So if $C$ is contracted in Proj $\oplus_{n \geqslant 0} \mathbb{C}\left[X^{\rho_{+}-s}\right]^{G, \rho_{0}^{n}}$, then it is also contracted in $\operatorname{Proj} \oplus_{n \geqslant 0} \mathbb{C}[X]^{G, \rho_{0}^{n}}$.

' $\Rightarrow$ ': Let the subgroup $G^{\prime} \subset G$ be the kernel of $\rho_{0}$. We show that there is a subvariety $P$ in $X^{\rho_{+}-s}$ satisfying

(a) $P$ is a $G^{\prime}$-principal bundle, and the base space is projective and connected;

(b) $F_{\rho_{+}}(P)=C$.

Here, $F_{\rho_{+}}$is the morphism $X^{\rho_{+}-s} \rightarrow X^{\rho_{+}-s} / / G$. Assume the existence of $P$ for the moment; then any function $f \in \mathbb{C}\left[X^{s, \rho_{+}}\right]^{G, \rho_{0}^{n}}$ is constant on each $G^{\prime}$-fiber. Since the base space is projective and connected, it must be constant on the whole subvariety $P$. Since $F_{\rho_{+}}(P)=C$, the value of $f$ on $F_{\rho_{+}}^{-1}(C)$ is determined by this constant. Hence, the canonical morphism contracts $C$ to a point.

To construct $P$, we may assume $G^{\prime} \neq G$. Choose $N$ large enough and finitely many $f_{i}$ in $\mathbb{C}[X]^{G, \rho_{0}^{N}}$ such that $\bigcap_{i}\left(V\left(f_{i}\right) \cap F_{\rho_{0}}^{-1}\left(\operatorname{pr}_{+}(C)\right)\right)$ is empty. Since all points in $F_{\rho_{0}}^{-1}\left(\operatorname{pr}_{+}(C)\right)$ are $S$-equivariant in $X^{\rho_{0}-s s}$, each $\overline{G x}$ contains all minimum orbits $G y$ in $F_{\rho_{0}}^{-1}\left(\operatorname{pr}_{+}(C)\right)$. Choose $y$ such that $G y$ is closed in $X^{\rho_{0}-s s}$, and let $P_{y}$ be

$$
\bigcap_{i}\left\{x \in F_{\rho_{+}}^{-1}(C) \mid f_{i}(x)=f_{i}(y)\right\} \text {. }
$$

For any $p \in C$, since the $G$-orbit $\overline{F_{\rho_{+}}^{-1}(p)}$ contains $y$ and $G$ is reductive, there are a subgroup $\beta: \mathbb{C}^{\times} \rightarrow G$ and $x_{p} \in F_{\rho_{+}}^{-1}(p)$ satisfying $y \in \overline{\beta\left(\mathbb{C}^{\times}\right) \times x_{p}}$. Since $y \in X^{\rho_{0}-s s}$, there is a $\rho_{0}^{N}$-semi-invariant $f_{i}$ such that $f_{i}(y)=0$. Therefore, $\rho_{0} \circ \beta=0$. This implies that for any $\rho_{0^{-}}$ semi-invariant function $f$, we have $f\left(x_{p}\right)=f(y)$, so Condition (b) is satisfied. Let $G^{\prime \prime}$ be the kernel of $\rho^{N}$. By the choices of the $f_{i}$, another point $x_{q}$ on $G x_{p}$ is in $P_{y}$ if and only if $x_{q}$ and $y$ are on the same $G^{\prime \prime}$-orbit. Since $G$ acts freely on all stable points, $P_{y}$ becomes a $G^{\prime \prime}$-principal bundle over the base $C$. As $\left[G^{\prime \prime}: G^{\prime}\right]$ is finite, we may choose a connected component of $P_{y}$ and as a $G^{\prime}$-principal bundle, the induced morphism from base space to $C$ is finite. Condition (a) is satisfied.

(v) This follows from [Tha96, Theorem 3.3].

Remark 5.3. When the difference between $X^{\rho_{+}-s}$ and $X^{\rho_{--}-s}$ is of codimension at least 2 in $X^{\rho_{+}, s} \cup X^{\rho_{-}-s}$, since $X^{\rho_{+}-s} \cup X^{\rho_{-}-s}$ is smooth, irreducible and quasi-affine by the second assumption, we have

$$
\mathbb{C}\left[X^{\rho_{+}-s}\right]^{G, \rho_{-}^{n}}=\mathbb{C}\left[X^{\rho_{+}-s} \cup X^{\rho_{-}-s}\right]^{G, \rho_{-}^{n}}=\mathbb{C}\left[X^{\rho_{-}-s}\right]^{G, \rho_{-}^{n}}=\mathbb{C}[X]^{G, \rho_{-}^{n}} \text { for } n \gg 0 .
$$

In this case, the birational map between $X^{\rho_{+}-s}$ and $X^{\rho_{-}-s}$ identifies $\mathrm{NS}_{\mathbb{R}}\left(X / / \rho_{+} G\right)$ and $\mathrm{NS}_{\mathbb{R}}\left(X / / \rho_{-}\right.$ $G)$. It maps $\left[\mathcal{L}_{\rho_{+}, \rho}\right]$ to $\left[\mathcal{L}_{\rho_{-}, \rho}\right]$ for any character $\rho$ in the chambers of both $\rho_{-}$and $\rho_{+}$. 


\section{MMP FOR DEFORMED HILB $\mathbb{P}^{2}$}

\subsection{Wall crossing in the second quadrant}

We will apply the results from previous sections to study the behavior of the moduli space of stable objects under wall crossing. In this section, we will focus on the case when the stability condition varies in the second quadrant of the $(s, t)$-plane. We will show that this is equivalent to the MMP of deformations of Hilbert scheme of points (Theorem 5.5). In particular, we get a concrete correspondence between the stable base locus walls in the effective cone and the actual destabilizing walls in the second quadrant (Proposition 5.7).

First, we need a lemma to make sure that the wall crossing eventually terminates in the second quadrant.

LEMma 5.4. There is a semicircular wall of radius greater than $\frac{1}{2}$ such that inside the wall, there is no semistable object with invariant $(1,0,1-n)$.

Proof. When $(k+2)(k+1)>2 n$, the $\ldots \mathcal{O}(-k)[1]$ always admits a non-zero morphism to any object $\mathcal{A}(-k)$ with dimension vector $\left(n_{-1}, n_{0}, n_{1}\right)=\left(n-(k-1) k / 2,2 n-k^{2}+1, n-(k+1) k / 2\right)$, since $2 n-k^{2}+1>3(n-(k+1) k / 2)$. When we look at the wall given by $\mathcal{O}(-k)[1]$, this is the wall containing $(-k, 0)$, and every object with invariant $(1,0,1-n)$ is destabilized at this wall. Hence, there is no stable object with invariant $(1,0,1-n)$ inside this semicircle.

Now we can state our main theorem.

THEOREM 5.5. When we vary the stability condition in the second quadrant of the $(s, t)$-plane of Bridgeland-stability conditions on $D^{b}(\mathrm{qgr}-S)$, the corresponding moduli space of stable objects performs birational transformations. This wall-crossing process is equivalent to the minimal model program for $\mathrm{Hilb}^{n} S$.

Proof. Each point in $\left\{(s, t) \mid-\sqrt{2 n}<s<0,0<t<\frac{1}{2}\right\}$ falls into at least one quiver region $\mathcal{A}(k)$. As explained before Proposition 3.7, the moduli space of $Z_{s, t}$-semistable objects with invariants $\left(r, c_{1}, \chi\right)=(1,0,1-n)$ is parameterized by the quotient space $X_{k} /\left.\right|_{\vec{\rho}_{s, t, k}} G_{k}$ (here, we put in the symbol $k$ to keep track of the quiver region). By Proposition 3.7, there are finitely many actual destabilizing walls, and in each chamber the moduli space remains the same. By the formula in Example 3.5 , the character $\vec{\rho}_{s, t, k}=\left(\rho_{-1}, \rho_{0}, \rho_{1}\right)$ always satisfies $\rho_{-1}>0>\rho_{1}$.

We first check that the $G_{k}$-variety $X_{k}$ satisfies the assumptions of Proposition 5.2 for all $\vec{\rho}_{s, t, k}$. Assumption (1), the 'finiteness of walls,' is due to the second property in Proposition 3.7. Assumptions (2), (3) and (4) follow from Theorems 4.3 and 4.11. Assumption (5) holds by King's criteria [Kin94] for (semi)stable quiver representations.

Now, for each point $(s, t)$, we may assign a divisor $\left[\mathcal{L}_{\overrightarrow{\rho_{+}, \vec{\rho}_{s, t, k}}}\right]$ to $X_{k} /\left.\right|_{\vec{\rho}_{s, t, k}} G_{k}$ as in Definition 5.1, where $\vec{\rho}_{+}$is a character in the chamber. Starting from a sufficient small $t>0$ and $-1<s<0$, where $X_{0} / \vec{\rho}_{s, t, 0} G_{0}$ is $\mathrm{Hilb}^{n} S$, fix $t$ and let $s$ decrease. At an actual destabilizing wall, let $\mathrm{pr}_{+}$ be the morphism from $X_{k} /\left.\right|_{\vec{\rho}_{s_{0}+\epsilon, t, k}} G_{k}$ to $X_{k} / \|_{\vec{\rho}_{s_{0}, t, k}} G_{k}$ as that in Proposition 5.2. One of three different cases may happen:

(1) The morphism $\mathrm{pr}_{+}$is a small contraction.

(2) The morphism $\mathrm{pr}_{+}$is birational and has an exceptional divisor.

(3) All points in $\mathrm{Hilb}^{n} S$ are destabilized.

In Case (1), by Proposition 4.10, we get small contractions on both sides of the wall. By property (v) in Proposition 5.2, this is the flip with respect to the divisor class $\left[\mathcal{L}_{\overrightarrow{\rho_{+}, \vec{\rho}_{s_{0}, t, k}}}\right]$. Since the loci of $X_{k}^{\rho_{+}-s}$ and $X_{k}^{\rho_{-}-s}$ which are strictly semistable on the wall are of codimension at 


\section{Li AND X. ZhaO}

most 2, their divisor cones can be identified as explained in Remark 5.3. When $s$ decreases, since the birational transformation that is performed is a flip, the divisor class $\left[\mathcal{L}_{\overrightarrow{\rho_{+}, \vec{\rho}_{s_{0}, t, k}}}\right]$ always jumps to the next chamber of the stable base locus decomposition of the pseudo-effective cone.

In Case (2), the morphism $X_{k} /\left\|_{\vec{\rho}_{s_{0}-\epsilon, t, k}} G_{k} \rightarrow X_{k} /\right\|_{\vec{\rho}_{s_{0}, t, k}} G_{k}$ does not have any exceptional divisors by Proposition 4.10; hence the Picard number of $X_{k} / \|_{\vec{\rho}_{0}-\epsilon, t, k} G_{k}$ is 1. By property (iv) in Proposition 5.2, Case (2) only happens when the canonical model associated with $\mathcal{L}_{\rho_{+}, \vec{\rho}_{s_{0}}, t, k}$ contracts a divisor, in other words, when the identified divisor of $\mathcal{L}_{\vec{\rho}_{+}, \vec{\rho}_{s, t, k}}$ on Hilb $S$ is on the boundary of the movable cone. When we continue decreasing $s$, the next destabilizing wall corresponds to the zero divisor, so it must be in Case (3). In general, if the boundary of the movable cone is not the same as the boundary of the nef cone, then Case (2) happens. Otherwise, Case (2) does not happen and the process terminates with a Mori fibration in Case (3).

Besides all previous ingredients, we only need to check that Case (3) happens before $s=-\sqrt{2 n}$ when $t=0+$. This is proved in Lemma 5.4, so we have completed our proof.

In particular, we get the following corollary. The special case of this for monomial schemes is also proved independently in [CH14].

Corollary 5.6. The semicircular actual walls in $W_{(1,0,1-n)}^{\text {act }}$ are in one-to-one correspondence with stable base locus decomposition walls on one side of the pseudo-effective cone of deformation of the Hilbert scheme of points Hilb ${ }^{n} S$.

In fact, we can make this correspondence more concrete. Given an integer $0 \leqslant k<\sqrt{2 n}$, for $-k-1<s<-k+1$, let $A_{k}$ and $B_{k}$ be the line bundles on $X_{-k} / \vec{\rho}_{s, 0+, k} G_{-k}$ whose transition functions are given by the composition of the transition functions of the $G$-principal bundle with characters $\left(2 n-k^{2}+1, k(k-1) / 2-n, 0\right)$ and $\left(0, n-k(k+1) / 2,-2 n+k^{2}-1\right)$, respectively. Then, when $-k-1<s<-k$, there are four divisors $A_{k}, B_{k}, A_{k+1}$ and $B_{k+1}$ on $X_{-k} / /_{\vec{\rho}_{s, 0+, k}} G_{-k}\left(\simeq X_{-k-1} / /_{\vec{\rho}_{s, 0+,-k-1}} G_{-k-1}\right)$ corresponding to the quiver regions $\mathcal{A}(-k)$ and $\mathcal{A}(-k-1)$. The line bundle $A_{k+1}$ as a line bundle on $X_{-k} /\left.\right|_{\vec{\rho}_{s, 0+,-k}} G_{-k}$ is with respect to the character $(n-k(k+1) / 2,0, k(k-1) / 2-n)$ up to a positive scalar. When the wall crossing in the quiver region only induces a flip, by Remark 5.3, these divisors satisfy the relation

$$
c_{k}\left[\begin{array}{l}
A_{k+1} \\
B_{k+1}
\end{array}\right]=\left[\begin{array}{cc}
2 n-k(k+1) & 2 n-k(k-1) \\
-2 n+(k+1)(k+2) & 3(2 n-(k-1)(k+2))
\end{array}\right]\left[\begin{array}{l}
A_{k} \\
B_{k}
\end{array}\right],
$$

where $c_{k}$ is a constant depending on $k$. In addition, $A_{k} \sim B_{k-2}$, where $\sim$ stands for equivalence up to a positive scalar. Let the first several line bundles be given as follows: $A_{1} \sim H, B_{0} \sim A_{2} \sim$ $(n-1) H-\frac{1}{2} \Delta, A_{3} \sim \frac{1}{2}(n-1) H-\frac{1}{2} \Delta$.

Proposition 5.7. The divisor corresponding to $(s, 0+)$ is $\left(-\left(2 n+s^{2}\right) / 2 s-3 / 2\right) H-\frac{1}{2} \Delta$ up to a scalar. In other words, the destabilizing semicircle wall on the Bridgeland-stability condition space with center $-m-\frac{3}{2}$ corresponds to the stable base locus decomposition wall $m H-\frac{1}{2} \Delta$.

Proof. Adopt the notation $A_{k}, B_{k}$ as above. We first show that when $k>0$, the divisors $A_{k}$ and $B_{k}$ are $(2 n+(k-1)(k-4)) H-(k-1) \Delta$ and $(2 n+(k-2)(k+1)) H-(k+1) \Delta$, respectively, up to a same scalar.

When $k=1$, we may assume $A_{1}=2 n H, B_{1}=b_{1}((n-1) H-\Delta)$ and $A_{2}=a_{2}\left((n-1) H-\frac{1}{2} \Delta\right)$. By the equation (5.1) for $k=1$, we have

$$
(2 n-2) A_{1}+2 n b_{1}\left(\frac{n-1}{2} H-\Delta\right) \sim(n-1) H-\frac{1}{2} \Delta .
$$




\section{MMP FOR DEFORMED HILB $\mathbb{P}^{2}$}

This implies $b_{1}=2$. By the equation (5.1) and induction on $k$, we have

$$
\begin{aligned}
{\left[\begin{array}{l}
A_{k+1} \\
B_{k+1}
\end{array}\right] } & \sim\left[\begin{array}{cc}
2 n-k(k+1) & 2 n-k(k-1) \\
-2 n+(k+1)(k+2) & 3(2 n-(k-1)(k+2))
\end{array}\right]\left[\begin{array}{c}
(2 n+(k-1)(k-4)) H-(k-1) \Delta \\
(2 n+(k-2)(k+1)) H-(k+1) \Delta
\end{array}\right] \\
& \sim\left[\begin{array}{c}
\left(8 n^{2}+(-6 k+2) 2 n-k\left(k^{2}-1\right)(2 k-6)\right) H-(4 n k-2(k-1) k(k+1)) \Delta \\
\left(8 n^{2}+(2 k-2) 2 n-\left(k^{2}-1\right)(k+2)(2 k-2)\right) H-\left(4 n(k+2)-2\left(k^{2}-1\right)(k+2)\right) \Delta
\end{array}\right] \\
& \sim\left(4 n-2\left(k^{2}-1\right)\right)\left[\begin{array}{c}
(2 n+k(k-3)) H-k \Delta \\
(2 n+(k+2)(k-1)) H-(k+2) \Delta
\end{array}\right],
\end{aligned}
$$

giving the formula for $A_{k+1}$ and $B_{k+1}$.

At the point $(s, 0+)$, the character $\rho_{s, 0+,-k}$ is given in Example 3.5. We have

$$
\rho_{s, 0+,-k}=-f(n, s, k-1)\left(0, \frac{n_{1}}{n_{0}},-1\right)+f(n, s, k+1)\left(1,-\frac{n_{-1}}{n_{0}}, 0\right),
$$

where $f(n, s, k)=k\left(2 n+s^{2}\right)+s\left(2 n+k^{2}\right)$. Up to a scalar, the divisor at $(s, 0+)$ is given by

$$
\begin{aligned}
-f(n, s, k-1) B_{k}+ & f(n, s, k+1) A_{k} \sim-f(n, s, k-1)((2 n+(k-2)(k+1)) H-(k+1) \Delta) \\
& \quad+f(n, s, k+1)((2 n+(k-4)(k-1)) H-(k-1) \Delta) \\
= & 2(2 n-(k-1)(k+1))\left(2 n+s^{2}+3 s\right) H \\
& +2 s(2 n-(k-1)(k+1)) \Delta \\
= & -2 s(2 n-(k-1)(k+1))\left(\left(-\frac{2 n+s^{2}}{2 s}-\frac{3}{2}\right) H-\frac{1}{2} \Delta\right) .
\end{aligned}
$$

The last statement follows directly from this formula.

\subsection{The vertical wall and wall crossing in the first quadrant}

In this section, we want to study the wall-crossing behavior across the vertical wall and walls in the first quadrant. In the non-deformed case, there is not much to say: when crossing the vertical wall, the Hilbert scheme of points maps to the symmetric product of $\mathbb{P}^{2}$, and this corresponds to the line bundle $H$. Note that the symmetric product is of Picard number 1 , so the next wall in the stable base locus decomposition is exactly given by $\Delta$, and the MMP terminates.

However, in the deformed case, the picture is much more interesting. We will see that crossing the vertical wall does not drop the Picard number of the moduli space of stable objects, and in the first quadrant there exists a sequence of wall-crossings 'symmetric' to that in the second quadrant.

Proposition 5.8. Given a Sklyanin algebra $\operatorname{Skl}(E, \mathcal{L}, \lambda)$, suppose that $\lambda$ is of infinite order; then no curve is contracted on the vertical wall $s=0$; in other words, the vertical wall is a fake wall.

Proof. The vertical wall corresponds to the wall in $\mathcal{A}(0)$ with respect to the character $(1,0,-1)$. We need the following criteria for stable monads.

Lemma 5.9. Suppose that the ... $\lambda$ of the Sklyanin algebra $\operatorname{Skl}(E, \mathcal{L}, \lambda)$ is of infinite order; then a monad $\mathbf{K}: \mathcal{O}(-1) \otimes \mathbb{C}^{n} \rightarrow \mathcal{O} \otimes \mathbb{C}^{2 n+1} \rightarrow \mathcal{O}(1) \otimes \mathbb{C}^{n}$ is stable with respect to $(1,0,-1)$ if and only if the first map is injective, the second map is surjective, and the homological sheaf $\mathrm{H}^{0}(\mathbf{K})$ at the middle term is a line bundle.

Proof. By the discussion in Proposition 3.7, the character $(1,0,-1)$ is on the wall of the Gieseker chamber that contains $((2 n+1)(m-1), n,-(2 n+1) m)$ for $m \gg 1$; hence, any $(1,0,-1)$ stable point is $((2 n+1)(m-1), n,-(2 n+1) m)$-stable and corresponds to a sheaf with invariant 


\section{Li AND X. ZhaO}

$\left(r, c_{1}, \chi\right)=(1,0,1-n)$. Denote by $I$ and $J$ the maps from $\mathcal{O}(-1) \otimes \mathbb{C}^{n}$ to $\mathcal{O} \otimes \mathbb{C}^{2 n+1}$ and from $\mathcal{O} \otimes \mathbb{C}^{2 n+1}$ to $\mathcal{O}(1) \otimes \mathbb{C}^{n}$, respectively. Write $I=x I_{1}+y I_{2}+z I_{3}$, where $I_{k}$ is a linear map from $\mathbb{C}^{n}$

to $\mathbb{C}^{2 n+1}$; then the monad corresponds to a line bundle if and only if the cokernel of $I$ is a vector bundle. By [NS07, Corollary 3.12 and Lemma 3.11] on the criteria of a vector bundle, $\mathrm{H}^{0}(\mathbf{K})$ is a line bundle if and only if $l I_{1}+m I_{2}+n I_{3}$ is injective for all non-zero triples $(l, m, n) \in \mathbb{C}^{3}$ (or equivalently for all $[(l, m, n)] \in E)$. Now, we may show the 'if' and 'only if' statements.

' $\Rightarrow$ ': Suppose that $\mathrm{H}^{0}(\mathbf{K})$ is not a line bundle; then $l I_{1}+m I_{2}+n I_{3}$ has a non-zero element $v_{-1}$ in its kernel. We may consider the minimum subcomplex $\mathbf{K}^{\prime}$ that contains $v_{-1}$. It is not hard to check that the dimension $\operatorname{dim}\left(H_{-1}^{\prime}, H_{0}^{\prime}, H_{1}^{\prime}\right)$ of $\mathbf{K}^{\prime}$ is $(1,0,0),(1,1,0),(1,2,0)$, or $(1,2,1)$. All of these cases contradict the $(1,0,-1)$-stability requirement.

' $\Leftarrow$ ': Suppose that the complex is not $(1,0,-1)$-stable; then a subcomplex with dimension vector $(a, b, c)$ destabilizes the monad. Since $\mathbf{K}$ is $((2 n+1)(m-1), n,-(2 n+1) m)$-stable for $m \gg 1$, we have $b \leqslant 2 a=2 c$. Restricting on the elliptic curve $E$, since $I$ is injective at every point, we have a complex on $E$ :

$$
0 \rightarrow \mathcal{L}^{*} \otimes \mathbb{C}^{a} \rightarrow \mathcal{O}_{E} \otimes \mathbb{C}^{b} \rightarrow \overline{\mathcal{L}} \otimes \mathbb{C}^{a} \rightarrow 0,
$$

which is exact except the middle term. Comparing the rank and the degree, we get $b=2 a$ and the complex is exact. Since $\lambda^{3 a}$ is not $\operatorname{id}_{E}$, we have $\mathcal{L}^{* \otimes a} \otimes \overline{\mathcal{L}}^{\otimes a} \nsucceq \mathcal{O}$. Therefore, the complex cannot be exact, which leads to the contradiction.

Proof of Proposition 5.8, continued. According to the proof of the lemma, any complex whose $\mathrm{H}^{0}(\mathbf{K})$ is not a line bundle has a subcomplex with dimension vector $(1,2,1)$. Hence, each $(1,0,-1)$-semistable complex has a filtration with $(1,0,-1)$-stable factors with the following dimension vectors: one copy of $(a, 2 a+1, a)$ (a line bundle $\mathcal{E}$ ) and several $(1,2,1)$ (quotient points $\mathcal{O}_{p}[-1]$ for $\left.p \in E\right)$.

Basic computations show that $\operatorname{Ext}^{1}\left(\mathcal{E}, \mathcal{O}_{p}[-1]\right)$ is $0, \operatorname{Ext}^{1}\left(\mathcal{O}_{p}[-1], \mathcal{E}\right)$ is $\mathbb{C}$ for all $p$ and $\operatorname{Ext}^{1}\left(\mathcal{O}_{p}[-1], \mathcal{O}_{q}[-1]\right)$ is $\mathbb{C}$ if and only if $p=q$ or $p=\lambda^{3}(q)$ and is 0 for any other $q$. Hence, the $\operatorname{Ext}^{1}$ of any two factors is at most 1 , and any $S$-equivariant class has only finitely many non-isomorphic complexes, which means that no curve is contracted.

Lemma 5.10. Let $X_{0}$ be the total space of complexes $\mathcal{O}(-1) \otimes \mathbb{C}^{n} \rightarrow \mathcal{O} \otimes \mathbb{C}^{2 n+1} \rightarrow \mathcal{O}(1) \otimes \mathbb{C}^{n}$, let $G_{0}$ be the group $\mathrm{GL}_{n} \times \mathrm{GL}_{2 n+1} \times \mathrm{GL}_{n} / \mathbb{C}^{\times}$, and let $\rho_{+}$be the character $(1,0,-1)+\epsilon(n,-2 n-1,0)$ for small enough positive $\epsilon$. Then $X_{0} / \rho_{\rho_{+}} G_{0}$ is smooth.

Proof. Given a stable complex $\mathbf{K}$ with respect to $\rho_{+}$, we may restricted it to the elliptic curve $E$. Since $\operatorname{Hom}\left(\mathbf{K}_{E}, \mathbf{K}_{E}\right)$ is $\mathbb{C}$, the hypercohomology of $\mathbf{H}^{2}\left(\mathcal{H o m} \bullet\left(\left.\mathbf{K}\right|_{E},\left.\mathbf{K}\right|_{E}\right)\right)$ is the same as $\operatorname{Ext}^{2}(\mathbf{K}, \mathbf{K})$. Since $\left.\mathbf{K}\right|_{E}$ is exact at the first term and the homological sheaf at the middle is a line bundle of non-positive degree, it is quasi-isomorphic to $\mathcal{Q} \rightarrow \overline{\mathcal{L}}^{\oplus n}$, where $\mathcal{Q}$ is locally free and $\mu_{+}(\mathcal{Q}) \leqslant 3=\mu(\overline{\mathcal{L}})$. Hence, $\mathbf{H}^{2}\left(\mathcal{H o m} \bullet\left(\left.\mathbf{K}\right|_{E},\left.\mathbf{K}\right|_{E}\right)\right)=0$. By a similar argument as that in Corollary 4.11, we deduce that $X / \rho_{\rho_{+}} G$ is smooth.

By Proposition 5.2(v), as no curve is contracted, we have a birational map $T_{w}: X_{0} / \rho_{\rho_{-}} G_{0}--\rightarrow$ $X_{0} / \rho_{\rho_{+}} G_{0}$, where $X / \rho_{\rho_{-}} G$ is $\operatorname{Hilb}^{n} S$. Since both varieties are smooth and $T_{w}$ does not have an exceptional locus, this is an isomorphism. Under this isomorphism, the line bundle complex remains the same (since these complexes are stable on both sides). Due to the uniqueness of the $S$-equivariant class, the $T_{w}$-image of an ideal complex $\mathcal{I}_{Z}$ with $Z$ equal to $n$ general distinct points $p_{1}, \ldots, p_{n}$ (by the term 'general,' we mean $\lambda^{3}\left(p_{i}\right) \neq p_{j}$ and $p_{i} \neq p_{j}$ for any $1 \leqslant i, j \leqslant n$ ) is shown below: 


\section{MMP FOR DEFORMED HILB $\mathbb{P}^{2}$}

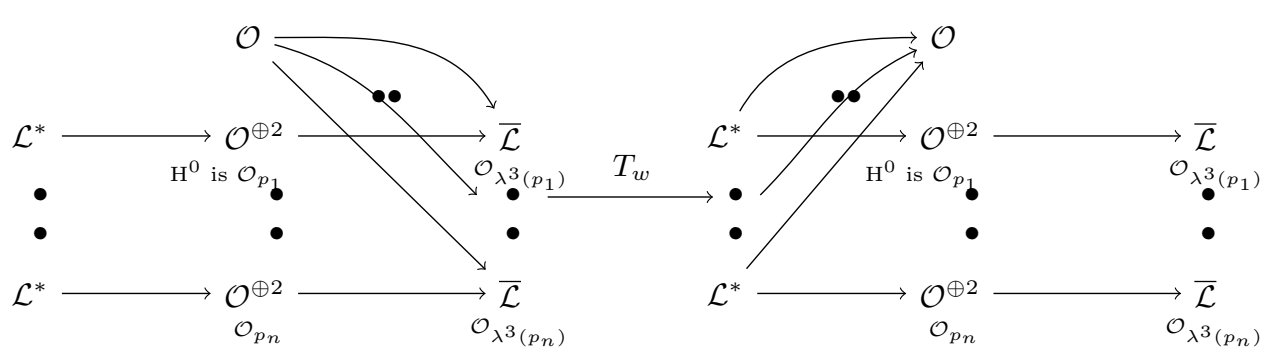

Write a complex $\mathbf{K}$ in $X_{0}^{s, \rho_{-}}$as $\mathcal{O}(-1) \otimes H_{-1} \stackrel{I}{\rightarrow} \mathcal{O} \otimes H_{0} \stackrel{J}{\rightarrow} \mathcal{O}(1) \otimes H_{1}$ with $I=x I_{1}+y I_{2}+z I_{3}$ and $J=x J_{1}+y J_{2}+z J_{3}$. Another morphism $\tilde{T}_{t}$ from $X_{0}^{s, \rho_{-}}$to $X_{0}^{s, \rho_{+}}$is defined as

$$
(I, J)=\left(x I_{1}+y I_{2}+z I_{3}, x J_{1}+y J_{2}+z J_{3}\right) \mapsto\left(x J_{2}^{T}+y J_{1}^{T}+z J_{3}^{T}, x I_{2}^{T}+y I_{1}^{T}+z I_{3}^{T}\right) .
$$

LEMma 5.11. The morphism $\tilde{T}_{t}$ is well defined and compatible with the $G_{0}$-action. In addition, it extends to other quiver regions as $\tilde{T}_{t, k}: X_{k}^{s, \rho_{-}} \rightarrow X_{-k}^{s, \rho_{+}}$.

Proof. Since $x, y, z$ satisfy the relations (2.1) in Definition 2.1, the image of a complex under $\tilde{T}_{t}$ is still a complex.

The stability property is due to duality. The image $\tilde{T}_{t}(\mathbf{K})$ is a complex $\mathcal{O}(-1) \otimes H_{1}^{*} \stackrel{I}{\rightarrow}$ $\mathcal{O} \otimes H_{0}^{*} \stackrel{J}{\rightarrow} \mathcal{O}(1) \otimes H_{-1}^{*}$. A subcomplex in $\tilde{T}_{t}(\mathbf{K})$ is determined by subspaces $\left(H_{1}^{\prime}, H_{0}^{\prime}, H_{-1}^{\prime}\right)$ in $\left(H_{1}^{*}, H_{0}^{*}, H_{-1}^{*}\right)$ that are compatible with $\tilde{T}_{t}(I, J)$. Then the subspaces $\left(H_{-1}^{\prime \perp}, H_{0}^{\prime \perp}, H_{1}^{\prime \perp}\right)$ in $\left(H_{-1}, H_{0}, H_{1}\right)$ are compatible with $I$ and $J$; hence, they determine a subcomplex of $\mathbf{K}$. Since $\rho_{+} \cdot\left(h_{1}^{\prime}, h_{0}^{\prime}, h_{-1}^{\prime}\right)>0$ if and only if $\rho_{-} \cdot\left(n_{-1}-h_{-1}^{\prime}, n_{0}-h_{0}, n_{1}-h_{1}\right)>0$, the complex $\tilde{T}_{t}(\mathbf{K})$ is $\rho_{+}$-stable. The morphism $\tilde{T}_{t, k}$ can be defined in the similar way. The compatibility of $\tilde{T}_{t, k}$ and $\tilde{T}_{t, k+1}$ is a routine check and is left to the reader.

As $\tilde{T}_{t}$ maps a $G_{0^{-}}$orbit to a $G_{0}$-orbit, it induces a map from $X_{0} / / \rho_{-} G_{0}$ to $X_{0} / \rho_{\rho_{+}} G_{0}$. We denote this isomorphism between $X^{s, \rho_{-}}$and $X^{s, \rho_{+}}$by $T_{t}$. This establishes the symmetry wall crossing picture between the first and second quadrants.

Denote by $T:=T_{t} \circ T_{w}$ the automorphism of $X_{0} / / \rho_{-} G_{0} \simeq \operatorname{Hilb}^{n} S$. By the definition of $T_{t}$, we have $T \circ T=\mathrm{Id}$. The following statement shows that when $n \geqslant 3$, the induced $T$-action on $\mathrm{NS}_{\mathbb{R}}\left(\mathrm{Hilb}^{n} S\right)$ is non-trivial; in other words, the destabilizing wall on the first quadrant destabilizes different points than the walls on the second quadrant.

Proposition 5.12. When $n \geqslant 3$, the automorphism $T$ on Hilb ${ }^{n} S$ induces a non-trivial action on $\mathrm{H}^{2}\left(\operatorname{Hilb}^{n} S, \mathbb{Z}\right)$.

Proof. When $n=3$, since the $\mathcal{O}(-1)$-wall (respectively, $\mathcal{O}(1)[1]$-wall) is the first wall on the left (respectively, right) of the $t$-axis, it is enough to show that these two walls destabilize different points on $X_{0} / \rho_{\rho_{+}} G_{0}$. To show this, we study when an ideal sheaf $\mathcal{I}_{Z}$ (that can be written as the kernel of $\mathcal{O} \rightarrow \oplus \mathcal{O}_{p_{i}}$ for three general distinct points $p_{1}, p_{2}, p_{3}$ on $E$ ) is destabilized on the $\mathcal{O}(-1)$-wall.

Let the complex of $\mathcal{I}_{Z}[1]$ be $\left(L^{*}\right)^{\oplus 3} \rightarrow \mathcal{O}_{E}^{\oplus 7} \rightarrow \overline{\mathcal{L}}^{\oplus 3}$, as the diagram on the left in (5.3). Write $\mathcal{E}$ for the kernel of $\mathcal{O}_{E}^{\oplus 7} \rightarrow \overline{\mathcal{L}}^{\oplus 3}$. As in (5.3), the map $\mathcal{O}_{E}^{\oplus 7} \rightarrow \overline{\mathcal{L}}^{\oplus 3}$ has four parts: $\mathcal{O}_{E}$ and three pieces $\mathcal{O}_{E}^{\oplus 2} \rightarrow \overline{\mathcal{L}}$. Each $\mathcal{O}_{E}^{\oplus 2} \rightarrow \overline{\mathcal{L}}$ has kernel $\overline{\mathcal{L}}^{-1}\left(\lambda^{3}\left(p_{i}\right)\right)$ and cokernel $\mathcal{O}_{\lambda^{3}\left(p_{i}\right)}$. The map from $\mathcal{E}$ to the direct sum of the three pieces $\mathcal{O}_{E}^{\oplus 2} \rightarrow \overline{\mathcal{L}}$ has kernel $\mathcal{O}\left(-\lambda^{3}\left(p_{1}\right)-\lambda^{3}\left(p_{2}\right)-\lambda^{3}\left(p_{3}\right)\right)$. 


\section{Li AND X. ZhaO}

Since the $\operatorname{Hom}\left(\mathcal{O}(-1), \mathcal{O}(i) \otimes \mathbb{C}^{n_{i}}\right)$ have dimensions $3,21,18$, for $i=-1,0,1$, respectively, we have the following equivalences:

$$
\begin{aligned}
& \operatorname{Hom}\left(\mathcal{O}(-1), \mathcal{I}_{Z}\right) \neq 0 \\
& \quad \Leftrightarrow \text { the map from } \operatorname{Hom}\left(\mathcal{O}(-1), \mathcal{O} \otimes \mathbb{C}^{7}\right) \text { to } \operatorname{Hom}\left(\mathcal{O}(-1), \mathcal{O}(1) \otimes \mathbb{C}^{3}\right) \text { is not surjective } \\
& \Leftrightarrow \text { the map from } \operatorname{Hom}\left(\mathcal{L}^{*}, \mathcal{O}_{E} \otimes \mathbb{C}^{7}\right) \text { to } \operatorname{Hom}\left(\mathcal{L}^{*}, \overline{\mathcal{L}} \otimes \mathbb{C}^{3}\right) \text { is not surjective } \\
& \Leftrightarrow \operatorname{Ext}^{1}\left(\mathcal{L}^{*}, \mathcal{E}\right) \neq 0 \\
& \Leftrightarrow \mathcal{O}\left(-\lambda^{3}\left(p_{1}\right)-\lambda^{3}\left(p_{2}\right)-\lambda^{3}\left(p_{3}\right)\right) \simeq \mathcal{L}^{*} .
\end{aligned}
$$

The last equivalence is due to the short exact sequence $0 \rightarrow \mathcal{O}\left(-\lambda^{3}\left(p_{1}\right)-\lambda^{3}\left(p_{2}\right)-\lambda^{3}\left(p_{3}\right)\right) \rightarrow$ $\mathcal{E} \rightarrow \oplus \overline{\mathcal{L}}^{-1}\left(\lambda^{3}\left(p_{i}\right)\right) \rightarrow 0$. A similar argument shows that $T_{w}\left(\mathcal{I}_{Z}[1]\right)$, whose diagram is on the right in (5.3), has non-zero morphism to $\mathcal{O}(1)[1]$ if and only if $\mathcal{O}\left(p_{1}+p_{2}+p_{3}\right) \simeq \overline{\mathcal{L}}$. Hence, $\mathcal{O}(-1)$ has non-zero morphism to $T\left(\mathcal{I}_{Z}\right)$ if and only if $\mathcal{O}\left(p_{1}+p_{2}+p_{3}\right) \simeq \overline{\mathcal{L}}$. Since $\overline{\mathcal{L}}\left(p_{i}\right)=\mathcal{L}^{*}\left(\lambda^{3}\left(p_{i}\right)\right)$ and $\lambda$ has infinite order, the locus that is contracted by the $\mathcal{O}(-1)$-wall and the locus that is contracted by the $\mathcal{O}(1)[1]$-wall are different.

When $n \geqslant 4$, we do induction on $n$. Assume that the $(n-1)$-case is done: a line bundle $\mathcal{I}$ with $\left(r, c_{1}, \chi\right)=(1,0,1-(n-1))$ is destabilized by $\mathcal{O}(-1)$, but $T(\mathcal{I})$ is not destabilized by $\mathcal{O}(-1)$. Consider the morphism $\mathcal{O}(-1) \rightarrow \mathcal{I}$ restricted to $E$; the cokernel is a torsion sheaf of length 3 . Let $\mathcal{O}_{p}$ be a quotient of the torsion sheaf; then $\mathcal{O}(-1)$ has a non-zero map to the kernel $\mathcal{I}^{\prime}$ of $\mathcal{I} \rightarrow \mathcal{O}_{p}$. Yet, $T\left(\mathcal{I}^{\prime}\right)$ is the kernel of $T(\mathcal{I}) \rightarrow \mathcal{O}_{q}$ for some $q \in E$, that is, $\operatorname{Hom}\left(\mathcal{O}(-1), T\left(\mathcal{I}^{\prime}\right)\right)=0$.

For any destabilize sequence $\mathcal{O}(-1) \rightarrow \mathcal{I} \rightarrow \mathcal{I}^{\prime \prime}$, the extension sheaf by $\mathcal{O}(-1)$ and $\mathcal{I}^{\prime \prime}$ is a vector bundle if and only if for any non-zero numbers $\left(l_{1}, l_{2}, l_{3}\right) \in \mathbb{C}^{3}$ on $E$, the $\ldots a I_{x}+b I_{y}+c I_{z}$ of $\mathcal{I}$ is injective, that is, $l_{1} I_{x}^{T}+l_{2} I_{y}^{T}+l_{3} I_{z}^{T}$ is surjective. For a generic choice of $\mathcal{I}^{\prime \prime}$, the cokernel of $x I_{x}^{\prime \prime T}+y I_{y}^{\prime \prime T}+z I_{z}^{\prime \prime T}$ restricted to $E$ is the direct sum of some skyscraper sheaves of distinct points. By adding an additive factor $\mathcal{O}(-1)$ at the middle term with suitable maps from $\mathcal{O}(-2) \otimes \mathbb{C}^{n_{-1}}$, $x I_{x}^{T}+y I_{y}^{T}+z I_{z}^{T}$ becomes surjective. Hence, on the loci that are destabilized by $\mathcal{O}(-1)$, the locus of line bundles is dense. Therefore, there exists a line bundle $\mathcal{V}$ that is destabilized by $\mathcal{O}(-1)$ while $T(\mathcal{V})$ is not destabilized by $\mathcal{O}(-1)$. This completes the induction step.

Combining Theorem 5.5 and Proposition 5.12, we get our main result on the generic deformed Hilbert scheme $\operatorname{Hilb}^{n} S$.

ThEOREM 5.13. When $n \geqslant 3$, the effective cone of $\operatorname{Hilb}^{n} S$ is symmetric. Stable base locus walls are in one-to-one correspondence with semicircular actual walls in both the first and the second quadrants of the $(s, t)$-plane of Bridgeland-stability conditions.

\section{Appendix. Sklyanin algebras and Bridgeland-stability conditions}

In this section, we prove several results on the Sklyanin algebra $S$ and Bridgeland-stability conditions on $D^{b}(\operatorname{qgr}-S)$. For the non-deformed case, that is, the projective plane, these are standard results.

Lemma A.1. Let $\mathcal{M} \in \operatorname{qgr}-S$ be a sheaf.

(i) We have $c_{1}(\mathcal{M}(s))=c_{1}(\mathcal{M})+s \cdot \operatorname{rk}(\mathcal{M})$ for any $s \in \mathbb{Z}$.

(ii) If $\mathcal{M}$ is torsion and non-zero, then $c_{1}(\mathcal{M}) \geqslant 0$; if, in addition, $c_{1}(\mathcal{M})=0$, then $\chi(\mathcal{M})>0$.

Proof. Property (i) is proved as the second property of Lemma 3.7 in [NS07]. 


\section{MMP FOR DEFORMED HiLb $\mathbb{P}^{2}$}

For property (ii), we can always find an integer $j$ such that there exists a non-zero morphism $\mathcal{O}_{S}(j) \rightarrow \mathcal{M}$. By the Noetherian hypothesis on $\mathcal{M}$, a quotient-descending chain of $\mathcal{M}$ is always finite. By the additivity of $c_{1}$ and $\chi$, we may assume that $\mathcal{O}_{S}(j) \rightarrow \mathcal{M}$ is surjective. To check that $c_{1}$ is non-negative, by the first property, we may assume $j=0$. Let $\mathcal{I}$ be the kernel of $\mathcal{O}_{S} \rightarrow \mathcal{M}$. Denote $\Gamma^{*}(\mathcal{I})$ by $I$. Write $c$ for $c_{1}(\mathcal{I})$; then $\mathcal{I}(-c)$ is a rank 1 , normalized (that is, $c_{1}(\mathcal{I}(-c))=0$ ), torsion-free sheaf. By [NS07, Proposition 5.6, Theorem 5.8, and Lemma 6.4], the shift $\mathcal{I}(-c)$ is the cohomology sheaf $\mathrm{H}^{0}(\mathbf{K})$ of the complex

$$
\mathbf{K}: \mathcal{O}_{S}(-1)^{\oplus a} \rightarrow \mathcal{O}_{S}^{\oplus 2 a+1} \rightarrow \mathcal{O}_{S}(1)^{\oplus a}
$$

at the middle term, where $a=1-\chi(\mathcal{I}(-c))$. As a graded module $I=\oplus_{n \in \mathbb{Z}} I_{n}$, and for $n \gg 0$, we have

$$
\begin{aligned}
\operatorname{dim}_{\mathbb{C}} I_{n} & =(2 a+1) \operatorname{dim}_{\mathbb{C}} S(c)_{n}-a \operatorname{dim}_{\mathbb{C}} S(c-1)_{n}-a \operatorname{dim}_{\mathbb{C}} S(c+1)_{n} \\
& =(2 a+1)\left(\begin{array}{c}
n+c+2 \\
2
\end{array}\right)-a\left(\left(\begin{array}{c}
n+c+1 \\
2
\end{array}\right)+\left(\begin{array}{c}
n+c+3 \\
2
\end{array}\right)\right) \\
& =\left(\begin{array}{c}
n+c+2 \\
2
\end{array}\right)-a .
\end{aligned}
$$

Since $\mathcal{I}$ is a subsheaf of $\mathcal{O}_{S}$, we have $\operatorname{dim}_{\mathbb{C}} I_{n}<\operatorname{dim}_{\mathbb{C}} S_{n}=\left(\begin{array}{c}n+2 \\ 2\end{array}\right)$ for $n \gg 0$. Since $a$ is a constant, the inequality holds for $n \gg 0$ if and only if $c \leqslant 0$; hence, $c_{1}(\mathcal{M}) \geqslant 0$.

When $\operatorname{rk}(\mathcal{M})=c_{1}(\mathcal{M})=0$, by [NS07][Lemma 6.1, Formula (2)], the Hilbert polynomial $p_{\mathcal{M}}(t)$ is a constant, so $\chi(\mathcal{M}(j))=\chi(\mathcal{M})$; we may also assume $j=0$. Then $\mathcal{I}$ is semistable and normalized, by [NS07, Lemma 6.4], we have $\chi(\mathcal{I}) \leqslant 1$, and equality holds only when $\mathcal{I}=\mathcal{O}$.

Recall that $D^{b}($ qgr- $S)$ is the bounded derived category of qgr- $S$. Here is one of the main results in [NS07], which is a non-commutative analog of the description of $D^{b}\left(\mathbb{P}^{2}\right)$ [Ber83].

Proposition A.2 ([NS07, Proposition 6.20]). The ... $D^{b}$ (qgr-S) is generated (that is, is the closure under extension and homological shift of $) \mathcal{O}(k-1), \mathcal{O}(k)$, and $\mathcal{O}(k+1)$ for any $k \in \mathbb{Z}$.

Proof. For any integer $m$, by induction and the exact sequence

$$
0 \rightarrow \mathcal{O}(m) \stackrel{\left[\begin{array}{l}
z \\
x \\
y
\end{array}\right]}{\longrightarrow} \mathcal{O}(m+1)^{\oplus 3} \stackrel{\left[\begin{array}{lll}
a y & c x & b z \\
b x & a z & c y \\
c z & b y & z x
\end{array}\right]}{\longrightarrow} \mathcal{O}(m+2)^{\oplus 3} \stackrel{\left[\begin{array}{l}
x \\
y \\
z
\end{array}\right]^{T}}{\longrightarrow} \mathcal{O}(m+3) \rightarrow 0,
$$

where $a, b, c$ are coefficients in $(2.1)$, we see that $\mathcal{O}(m)$ is in the closure for any integer $m$. By [NS07, Proposition 6.20], all semistable sheaves are in the closure. Since each torsion-free sheaf admits a finite Harder-Narasimhan filtration and each torsion sheaf is the cokernel of a morphism between two torsion free sheaves, all sheaves are in the closure.

Now, we can generalize the construction of Bridgeland-stability conditions on $D^{b}\left(\mathbb{P}^{2}\right)$ to $D^{b}(\operatorname{qgr}-S)$.

Proposition A.3. Adopt the notation from Section 2.2; the pair $\left(Z_{s, t}, \mathrm{Coh}_{\# s}\right)$ is a stability condition on $D^{b}(\operatorname{qgr}-S)$.

Proof. Any object $E \in \mathrm{Coh}_{\# s}$ fits in an exact sequence

$$
0 \rightarrow \mathrm{H}^{-1}(E)[1] \rightarrow E \rightarrow \mathrm{H}^{0}(E) \rightarrow 0 .
$$




\section{Li AND X. ZhaO}

In order to check the first property of the central charge in Definition 2.6, we only need to check that we have $\operatorname{Arg}\left(Z_{s, t}(E)\right) \in(0, \pi]$ for the following cases: 1) $E$ is a torsion sheaf; 2) $E$ is a slope semistable sheaf with $\mu(E)>s$; 3) $E[-1]$ is a slope semistable sheaf with $\mu(E[-1]) \leqslant s$.

Case 1 is a consequence of Lemma A.1. Case 2 is clear since $r_{s, t}(E)$ is greater than 0 .

In Case 3, we may assume $c_{1}(E[-1])=r(E[-1]) s$, since in other cases $r_{s, t}(E)>0$. Then

$$
d_{s, t}(E)=\frac{r t^{2}}{2}+r+\frac{3 c_{1}}{2}-\chi+\frac{c_{1}^{2}}{2 r} \geqslant \frac{r t^{2}}{2}+\frac{r^{2}-1}{2 r}>0,
$$

where $r, c_{1}, \chi$ stand for $r(E[-1]), c_{1}(E[-1]), \chi(E[-1])$, respectively. The first inequality is due to the following non-commutative version of the Bogomolov inequality.

Lemma A.4 ([NS07, Corollary 6.2 and Proposition 2.4]). For a slope semistable sheaf $E$ in qgr-S, we have $2 \chi r-r^{2}-3 r c_{1}-c_{1}^{2} \leqslant 1$.

To see this, just notice

$$
\begin{aligned}
2 \chi r-r^{2}-3 r c_{1}-c_{1}^{2} & =\chi(E[-1], E[-1]) \leqslant 1+\operatorname{ext}^{2}(E[-1], E[-1]) \\
& =1+\operatorname{hom}(E[-1], E[-1](-3))=1 .
\end{aligned}
$$

Proof of Proposition A.3, continued (1). In Case 3, we thus see that $\operatorname{Arg}\left(Z_{s, t}(E)\right)=\pi$; hence, we have proved the first property of the central charge in Definition 2.6.

Remark A.5. Lemma A.4 implies the formal Bogomolov inequality for slope semistable sheaf $E$ :

$$
Q:=c_{1}^{2}-2 \operatorname{ch}_{2} r \geqslant r^{2}-1 \geqslant 0 .
$$

Proof of Proposition A.3, continued (2). To prove the Harder-Narasimhan (HN) property, we need the following lemma to check the descending chain condition.

Lemma A.6. For a pair $\left(Z_{s, t}, \mathrm{Coh}_{\# s}\right)$ and two positive numbers $M_{1}, M_{2}$, the set

$$
\left\{\left(-d_{s, t}, r_{s, t}\right) \mid-d_{s, t}(\mathcal{F}) \leqslant M_{1}, 0 \leqslant r_{s, t}(\mathcal{F}) \leqslant M_{2}, \mathcal{F} \in \mathrm{Coh}_{>s} \text { and is a torsion-free sheaf }\right\}
$$

is finite.

Proof. First, we show that this holds for all slope semistable sheaves. Write $\chi(\mathcal{F}), r(\mathcal{F}), c_{1}(\mathcal{F})$ as $\chi, r, c_{1}$ for short. By Lemma A.4, we have

$$
\chi \leqslant \frac{1}{2 r}\left(1+r^{2}+3 r c_{1}+c_{1}^{2}\right) .
$$

Substituting this inequality into the formula of $d_{s, t}$, we have

$$
-d_{s, t} \geqslant \frac{r\left(t^{2}+1\right)}{2}-\frac{\left(c_{1}-r s\right)^{2}}{2 r}-\frac{1}{2 r} .
$$

Since $c_{1}-r s \in\left[0, M_{2}\right]$ and $M_{1} \geqslant-d_{s, t}$, we see that $r$ is bounded. As $c_{1}-r s \in\left[0, M_{2}\right]$, it follows that $c_{1}$ is bounded. Now, by the inequality of $\chi$ and the formula of $d_{s, t}$, we have

$$
-M_{1}+\frac{r t^{2}}{2}-\left(\frac{s^{2}}{2}-1\right) r-\left(\frac{3}{2}+s\right) c_{1} \leqslant \chi \leqslant \frac{1}{2 r}\left(1+r^{2}+3 r c_{1}+c_{1}^{2}\right) .
$$

Hence, $\chi$ is bounded. The set

$\left\{\left(-d_{s, t}, r_{s, t}\right) \mid-d_{s, t}(\mathcal{F}) \leqslant M_{1}, 0 \leqslant r_{s, t}(\mathcal{F}) \leqslant M_{2}, \mathcal{F}\right.$ is in $\mathrm{Coh}_{\# s}$ and is a slope semistable sheaf $\}$ is finite. 


\section{MMP FOR DEFORMED HILB $\mathbb{P}^{2}$}

Next, we show that this holds for any torsion-free sheaf $F \in \mathrm{Coh}_{\# s}$. By the finiteness result above, we may define

$$
\begin{aligned}
D & :=\min \left\{-d_{s, t}(\mathcal{F}) \mid \mathcal{F} \text { is in } \operatorname{Coh}_{\# s} \text { and is a torsion-free slope semistable sheaf }\right\} \\
R & :=\min \left\{r_{s, t}(\mathcal{F}) \mid \mathcal{F} \text { is in } \operatorname{Coh}_{\# s} \text { and is a torsion-free slope semistable sheaf }\right\} .
\end{aligned}
$$

Now given a torsion free sheaf $\mathcal{G} \in \mathrm{Coh}_{\# s}$, for each HN factor $\mathcal{G}_{i}$ of $G$, we see that $-d_{s, t}\left(\mathcal{G}_{i}\right)<$ $(1 / R) D^{2}+M_{1}$.

Therefore,

$$
\begin{aligned}
& \left\{\left(-d_{s, t}, r_{s, t}\right) \mid-d_{s, t}(\mathcal{F}) \leqslant M_{1}, 0 \leqslant r_{s, t}(\mathcal{F}) \leqslant M_{2}, \mathcal{F} \text { is in } \mathrm{Coh}_{\# s} \text { and is a torsion-free sheaf }\right\} \\
& \subset \sum\left\{\left(-d_{s, t}, r_{s, t}\right) \mid-d_{s, t}(\mathcal{G}) \leqslant \frac{D^{2}}{R}+M_{1}, 0 \leqslant r_{s, t}(\mathcal{G}) \leqslant M_{2},\right. \\
& \left.\mathcal{G} \in \mathrm{Coh}_{\# s} \text { and is a slope semistable sheaf }\right\} .
\end{aligned}
$$

Now, in the second set, $r_{s, t}>0$, so by the finiteness, $r_{s, t}$ is bounded from below by a positive constant. As a result, the number of summands in the second set is finite; hence, we conclude the finiteness of the first set.

Proof of Proposition A.3, continued (3). Now, we may check the descending chain condition. Suppose that the condition does not hold; then we have an object $E$ in $\mathcal{A}_{s}$ that has an infinite descending chain

$$
\cdots \subset E_{i+1} \subset E_{i} \subset \cdots \subset E_{1} \subset E_{0}=E
$$

with strictly increasing slopes $\mu_{s, t}\left(E_{i+1}\right)>\mu_{s, t}\left(E_{i}\right)$ for all $i$. There are short exact sequences in $\mathrm{Coh}_{\# s}: 0 \rightarrow E_{i+1} \rightarrow E_{i} \rightarrow F_{i} \rightarrow 0$ for $i \geqslant 0$. By taking the cohomology sheaves, we have $\mathrm{H}^{-1}\left(E_{i+1}\right) \subset \mathrm{H}^{-1}\left(E_{i}\right)$. We may assume that the rank of $\mathrm{H}^{-1}\left(E_{i}\right)$ is constant. Now, the cokernel $\mathrm{H}^{-1}\left(E_{i}\right) / \mathrm{H}^{-1}\left(E_{i+1}\right)$ must be torsion, but $\mathrm{H}^{-1}\left(F_{i}\right)$ is torsion free, so we have $\mathrm{H}^{-1}\left(E_{i}\right) \simeq$ $\mathrm{H}^{-1}\left(E_{i+1}\right)$.

Let $T_{i}$ and $G_{i}$ be the torsion subsheaf and torsion-free quotient of $\mathrm{H}^{0}\left(E_{i}\right)$, respectively. Since we have the exact sequence

$$
0 \rightarrow \mathrm{H}^{-1}\left(F_{i}\right) \rightarrow \mathrm{H}^{0}\left(E_{i+1}\right) \rightarrow \mathrm{H}^{0}\left(E_{i}\right) \rightarrow \mathrm{H}^{0}\left(F_{i}\right) \rightarrow 0,
$$

and $\mathrm{H}^{-1}\left(F_{i}\right)$ is torsion free, $T_{i+1}$ is a subsheaf of $T_{i}$. We may assume that $c_{1}\left(T_{i}\right)$ is constant; then $\chi\left(T_{i}\right)$ is non-increasing.

Combining all the above assumptions, we have

$$
\begin{gathered}
-d_{s, t}\left(\mathrm{H}^{-1}\left(E_{i}\right)[1]\right)-d_{s, t}\left(T_{i}\right) \geqslant-d_{s, t}\left(\mathrm{H}^{1}\left(E_{0}\right)[1]\right)-d_{s, t}\left(T_{0}\right), \\
r_{s, t}\left(\mathrm{H}^{-1}\left(E_{i}\right)[1]\right)+r_{s, t}\left(T_{i}\right) \geqslant r_{s, t}\left(\mathrm{H}^{1}\left(E_{0}\right)[1]\right)+r_{s, t}\left(T_{0}\right) .
\end{gathered}
$$

On the other hand, since the slope is increasing, we also have

$$
-d_{s, t}\left(E_{i}\right) \leqslant \max \left\{-d_{s, t}\left(E_{0}\right), 0\right\}, \quad r_{s, t}\left(E_{i}\right) \leqslant r_{s, t}\left(E_{0}\right) .
$$

Subtracting the first set of inequalities from the second, we have

$$
-d_{s, t}\left(G_{i}\right) \leqslant \max \left\{-d_{s, t}\left(G_{0}\right), d_{s, t}\left(\mathrm{H}^{-1}\left(E_{0}\right)[1]\right)+d_{s, t}\left(T_{0}\right), 0\right\}, \quad r_{s, t}\left(G_{i}\right) \leqslant r_{s, t}\left(G_{0}\right) .
$$

Now, applying Lemma A.6 to $G_{i}$ and combining with the results on $\mathrm{H}^{-1}\left(E_{i}\right)[1]$ and $T_{i}$, we see that the set of possible values of $\left(-d_{s, t}\left(E_{i}\right), r\left(E_{i}\right)\right)$ is finite, so we obtain the descending chain condition. 


\section{Li AND X. ZhaO}

The ascending chain condition can be proved similarly, where one applies Lemma A.6 to $\mathrm{H}^{-1}\left(E_{i}\right)[1]$ to get the finiteness. In this case, the area in the lemma becomes $\left[-M_{1},+\infty\right) \times\left[0, M_{2}\right]$ because of the homological shift. The rest of the argument goes similarly to that above, and the details are left to the readers.

Finally, we show the support condition by showing that the quadratic form $Q$ as defined in Remark A.5 satisfies the conditions in Definition 2.7 for any $(t, s) \in \mathbb{R}_{>0} \times \mathbb{R}$. For the first condition, note that the kernel of $Z_{s, t}$ in $K(\operatorname{qgr}-S) \otimes \mathbb{R}$ is the line $\left(r, c_{1}, \mathrm{ch}_{2}\right)=\left(r, r s, \frac{1}{2}\left(r s^{2}+r t^{2}\right)\right)$. On the kernel, we have $Q=(r s)^{2}-\left(r s^{2}+r t^{2}\right) r=-r^{2} t^{2} \leqslant 0$. Now, we only need to show that for any $\sigma_{s, t}$-stable object $E$ in $\operatorname{Coh}_{\# s}$, we have $Q(v(E)) \geqslant 0$.

Suppose $Q(v(E))<0$; in particular, $r(E)$ is not 0 and $E$ is not slope semistable.

If $r(E)>0$, then $\mathrm{H}^{0}(E)$ is non-zero. Let $F=\mathrm{H}^{0}(E)_{\text {min }}$ be the quotient sheaf of $\mathrm{H}^{0}(E)$ with the minimum slope. Let $D$ be $\mathrm{H}^{-1}(E)$, and let $G$ be the kernel of $\mathrm{H}^{0}(E) \rightarrow F$. We may compare the slopes of $D$ and $G: \mu(D)<\mu(F)<\mu(G)$ when $D$ and $G$ are non-zero. We have the relation

$$
\frac{c_{1}(E)}{r(E)}=\frac{c_{1}(F)+c_{1}(G)-c_{1}(D)}{r(F)+r(G)-r(D)} \geqslant \frac{c_{1}(F)}{r(F)} .
$$

Equality holds only when $D$ and $G$ are both zero, but this is not possible as else $v(E)=v(F)$ and $Q(v(E)) \geqslant 0$ by Remark A.5. Therefore, we have $s \leqslant c_{1}(F) / r(F)<c_{1}(E) / r(E)$. Since $E$ is $\sigma_{s, t}$-stable, we have $\mu_{s, t}(E) \leqslant \mu_{s, t}(F)$. As $s \leqslant c_{1}(F) / r(F)<c_{1}(E) / r(E)$, the inequality $\mu_{s, t}(E) \leqslant \mu_{s, t}(F)$ is equivalently to saying that the point $\left(c_{1}(F) / r(F), \operatorname{ch}_{2}(F) / r(F)\right)$ is above the line segment connecting $\left(s, \frac{1}{2}\left(s^{2}+t^{2}\right)\right)$ and $\left(c_{1}(E) / r(E), \operatorname{ch}_{2}(E) / r(E)\right)$. Since both points $\left(s, \frac{1}{2}\left(s^{2}+t^{2}\right)\right)$ and $\left(c_{1}(E) / r(E), \mathrm{ch}_{2}(E) / r(E)\right)$ are above the parabola $y=\frac{1}{2} x^{2}$ and the parabola is convex up, the point $\left(c_{1}(F) / r(F), \operatorname{ch}_{2}(F) / r(F)\right)$ is also above the parabola. Therefore, we have $\frac{1}{2}\left(c_{1}(F) / r(F)\right)^{2}<\operatorname{ch}_{2}(F) / r(F)$, or $Q(v(F))<0$, which contradicts Remark A.5.

When $r(E)<0$, let $F=\mathrm{H}^{-1}(E)_{\max }$ be the subsheaf of $\mathrm{H}^{-1}(E)$ with maximum Mumford slope. By a similar argument, we have $c_{1}(E) / r(E)<c_{1}(F) / r(F) \leqslant s$ and the point $\left(c_{1}(F) / r(F), \operatorname{ch}_{2}(F) / r(F)\right)$ is above the line segment connecting the points $\left(s, \frac{1}{2}\left(s^{2}+t^{2}\right)\right)$ and $\left(c_{1}(E) / r(E), \operatorname{ch}_{2}(E) / r(E)\right)$. Due to the same argument, we have $Q(v(F))<0$ and get the contradiction.

\section{ACKNOWLEDGEMENTS}

We are first of all greatly indebted to Emanuele Macrì, who offered tremendous assistance during the preparation of this work. We are grateful to Arend Bayer, Aaron Bertram, Izzet Coskun, Jack Huizenga and Cristian Martinez for helpful conversations. We also had useful discussions with our advisors Herbert Clemens, Robert Lazarsfeld, Thomas Nevins and Karen Smith, and we would like to thank all of them.

\section{REFERENCES}

AB13 D. Arcara and A. Bertram, Bridgeland-stable moduli spaces for K-trivial surfaces (with an appendix by Max Lieblich), J. Eur. Math. Soc. (JEMS) 15 (2013), no. 1, 1-38; https://doi. org/10.4171/JEMS/354.

ABCH13 D. Arcara, A. Bertram, I. Coskun, and J. Huizenga, The minimal model program for the Hilbert scheme of points on $\mathbb{P}^{2}$ and Bridgeland stability, Adv. Math. 235 (2013), 580-626; https://doi.org/10.1016/j.aim.2012.11.018. 


\section{MMP FOR DEFORMED HILB $\mathbb{P}^{2}$}

ATVdB90 M. Artin, J. Tate, and M. van den Bergh, Some algebras associated to automorphisms of elliptic curves, The Grothendieck Festschrift, Vol. I, Progr. Math., vol. 86 (Birkhäuser Boston, Boston, MA, 1990), 33-85; https://doi.org/10.1007/978-0-8176-4574-8_3.

ATVdB91 , Modules over regular algebras of dimension 3, Invent. Math. 106 (1991), no. 2, 335388; https://doi.org/10.1007/BF01243916.

AVdB90 M. Artin and M. van den Bergh, Twisted homogeneous coordinate rings, J. Algebra 133 (1990), no. 2, 249-271; https://doi.org/10.1016/0021-8693(90)90269-T.

BC13 A. Bertram and I. Coskun, The birational geometry of the Hilbert scheme of points on surfaces, Birational Geometry, Rational Curves, and Arithmetic (Springer, New York, 2013), 15-55; https://doi.org/10.1007/978-1-4614-6482-2_2.

Beř83 A. A Beylinson, The derived category of coherent sheaves on $\mathbf{P}^{n}$, Selecta Math. Soviet. 3 (1983), no. 3, 233-237.

BM14a A. Bayer and E. Macrì, MMP for moduli of sheaves on K3s via wall-crossing: nef and movable cones, Lagrangian fibrations, Invent. Math. 198 (2014), no. 3, 505-590; https://doi.org/ 10.1007/s00222-014-0501-8.

BM14b_, Projectivity and birational geometry of Bridgeland moduli spaces, J. Amer. Math. Soc. 27 (2014), no. 3, 707-752; https://doi.org/10.1090/S0894-0347-2014-00790-6.

BMS16 A. Bayer, E. Macrì, and P. Stellari, The space of stability conditions on abelian threefolds, and on some Calabi-Yau threefolds, Invent. Math. 206 (2016), no. 3, 869-933; https://doi.org/ 10.1007/s00222-016-0665-5.

Bri07 T. Bridgeland, Stability conditions on triangulated categories, Ann. of Math. 166 (2007), no. 2, 317-345; https://doi.org/10.4007/annals.2007.166.317.

Bri08_ Stability conditions on K3 surfaces, Duke Math. J. 141 (2008), no. 2, 241-291; https: //doi.org/10.1215/S0012-7094-08-14122-5.

CH14 I. Coskun and J. Huizenga, Interpolation, Bridgeland stability and monomial schemes in the plane, J. Math. Pures Appl. 102 (2014), no. 5, 930-971; https://doi.org/10.1016/ j.matpur.2014.02.010.

CHW17 I. Coskun, J. Huizenga, and M. Woolf, The effective cone of the moduli space of sheaves on the plane, J. Eur. Math. Soc. 19 (2017), no. 5, 1421-1467; https://doi .org/10.4171/JEMS/696.

Del77 P. Deligne, Cohomologie étale. Séminaire de Géométrie Algébrique du Bois-Marie SGA 4 1/2, Lecture Notes in Math., vol. 569 (Springer-Verlag, Berlin - New York, 1977); https://doi. org/10.1007/BFb0091516.

DH98 I. V. Dolgachev and Y. Hu, Variation of geometric invariant theory quotients (with an appendix by Nicolas Ressayre), Publ. Math. Inst. Hautes Études Sci. (1998), no. 87, 5-56; https: //doi.org/10.1007/BF02698859.

GD67 A. Grothendieck and J. Dieudonné, Éléments de géométrie algébrique. IV. Étude locale des schémas et des morphismes de schémas. IV, Publ. Math. Inst. Hautes Études Sci. 32 (1967), 5-333; https://doi.org/10.1007/BF02732123.

Gin12 V. Ginzburg, Lectures on Nakajima's quiver varieties, Geometric Methods in Representation Theory. I, Sémin. Congr., vol. 24 (Soc. Math. France, Paris, 2012), 145-219.

Hit12 N. Hitchin, Deformations of holomorphic Poisson manifolds, Mosc. Math. J. 12 (2012), no. 3, 567-591; http://www.ams.org/distribution/mmj/vol12-3-2012/hitchin.pdf.

Kin94 A. D. King, Moduli of representations of finite-dimensional algebras, Quart. J. Math. Oxford Ser. (2) 45 (1994), no. 180, 515-530; https://doi.org/10.1093/qmath/45.4.515.

KS08 M. Kontsevich and Y. Soibelman, Stability structures, motivic Donaldson-Thomas invariants and cluster transformations, 2008, arXiv:0811.2435.

Mac07 E. Macrì, Stability conditions on curves, Math. Res. Lett. 14 (2007), no. 4, 657-672; https : //doi.org/10.4310/MRL.2007.v14.n4.a10. 


\section{LI AND X. ZHAO}

Mac14 A. Maciocia, Computing the walls associated to Bridgeland stability conditions on projective surfaces, Asian J. Math. 18 (2014), no. 2, 263-279; https://doi.org/10.4310/AJM.2014. v18.n2.a5.

MS16 E. Macrì and B. Schmidt, Lectures on Bridgeland stability, 2016, arXiv:1607.01262.

MYY11 H. Minamide, S. Yanagida, and K. Yoshioka, Fourier-Mukai transforms and the wall-crossing behavior for Bridgeland's stability conditions, 2011, arXiv:1106.5217.

MYY14 H. Minamide, S. Yanagida, and K. Yoshioka, Some moduli spaces of Bridgeland's stability conditions, Int. Math. Res. Not. 2014 (2014), no. 19, 5264-5327; https ://doi .org/10.1093/ imrn/rnt126.

Nak97 H. Nakajima, Heisenberg algebra and Hilbert schemes of points on projective surfaces, Ann. of Math. 145 (1997), no. 2, 379-388; https://doi.org/10.2307/2951818.

NS07 T. A. Nevins and J. T. Stafford, Sklyanin algebras and Hilbert schemes of points, Adv. Math. 210 (2007), no. 2, 405-478; https://doi.org/10.1016/j.aim.2006.06.009.

Nue16 H. Nuer, Projectivity and birational geometry of Bridgeland moduli spaces on an Enriques surface, Proc. Lond. Math. Soc. 113 (2016), no. 3, 345-386; https://doi.org/10.1112/ plms/pdw033.

SvdB01 J. T. Stafford and M. van den Bergh, Noncommutative curves and noncommutative surfaces, Bull. Amer. Math. Soc. (N.S.) 38 (2001), no. 2, 171-216; https://doi.org/10.1090/ S0273-0979-01-00894-1.

Tha96 M. Thaddeus, Geometric invariant theory and flips, J. Amer. Math. Soc. 9 (1996), no. 3, 691-723; https://doi.org/10.1090/S0894-0347-96-00204-4.

Chunyi Li C.Li.25@warwick.ac.uk

School of Mathematics, The University of Edinburgh, James Clerk Maxwell Building, The King's Buildings, Mayfield Road, Edinburgh, Scotland EH9 3JZ, United Kingdom

Current address: Mathematics Institute, University of Warwick, Coventry, CV4 7AL, United Kingdom

Xiaolei Zhao X.Zhao@neu.edu

Department of Mathematics, Northeastern University, 360 Huntington Avenue, Boston, MA 02115, USA 